ANNALES

UNIVERSITATIS MARIAE C URIE-SKŁODOW K A LUBLIN - POLONIA

VOL. LXXI, 2
SECTIO C 2016

\title{
ANETA CZARNA
}

Department of Botany

Poznań University of Life Sciences

ul. Wojska Polskiego 71c, 60-625 Poznań, Poland; e-mail: czarna@up.poznan.pl

\author{
Roses (Rosa spp.) in old cemeteries \\ in the Wielkopolska region (W Poland)
}

\section{SUMMARY}

This paper presents all taxa of the genus Rosa recorded in 2005-2015 in old cemeteries in Wielkopolska. In the past, roses were frequently planted in cemeteries as ornamentals, contributing to their beauty and specific atmosphere, but they also had many symbolic meanings. Today, historical roses are rare. As a result of floristic research in 2,169 old, neglected cemeteries in Wielkopolska, 35 taxa of roses were found. These include 21 alien taxa and 6 taxa new to Poland.

Keywords: Rosa, flora, cemeteries, chorology, symbolic meaning, Wielkopolska, Poland

\section{STRESZCZENIE}

Praca przedstawia wszystkie gatunki Rosa stwierdzone w latach 2005-2015 na starych cmentarzach Wielkopolski. W przeszłości gatunki róż chętnie wysadzano na cmentarzach jako rośliny ozdobne, nadając miejscu uroku oraz specyficznej atmosfery. Miały także wielorakie znaczenie symboliczne. Dziś róże historyczne należą już do rzadkości. Na przebadanych florystycznie 2169 starych nieczynnych cmentarzach z terenu Wielkopolski stwierdzono 35 taksonów róż, wśród których 21 to taksony obce dla flory Polski. Odnotowano sześć taksonów nowych dla Polski.

Słowa kluczowe: Rosa, flora, cmentarze, chorologia, symbolika, Wielkopolska, Polska

\section{INTRODUCTION}

Old, neglected cemeteries are interesting from the botanical point of view. Some of the plants found there are nearly completely absent from new cemeteries. These include also old, forgotten taxa of roses (historical roses). 
The major asset of historical roses is that they are romantic, single- or double-flowered, sometimes even with pompom flowers, which delight rose lovers. The flowers have an exceptionally varied, captivating palette of colours and shades, and very pleasant smell. However, old roses have 3 basic colours: white, pink, and red, with few exceptions. Roses are generally characterized by stolons, spreading easily. In the garden this can be very troublesome, but in the wild it may be a desirable feature.

As early as the Middle Ages, roses made a stunning career as plants used for ornamenting of burial sites and other places commemorating the dead. The fact that they were brought from the Holy Land by crusaders was a sufficient recommendation at that time [Internet 1]. For many centuries, rose species and varieties were frequently planted in cemeteries as ornamentals, contributing to their beauty and specific atmosphere, but also had many symbolic meanings.

Spontaneously occurring roses were investigated in nearly all parts of the Wielkopolska region (11, 16), and the Lubusz Land (Ziemia Lubuska) (15), but old cemeteries were not taken into account in those studies. This paper presents a list of localities (=cemeteries) of Rosa taxa found in Wielkopolska in 2005-2015 as a result of investigations in old cemeteries.

\section{CHARACTERISTICS OF ROSES}

The genus Rosa globally comprises about 120 species, found exclusively in the Northern Hemisphere, including about 50 species in Europe (8). In Poland in the wild, 25 species are found, but 11 of them are naturalized alien species (17), while 16 are native (7). Roses are distributed worldwide, primarily in the temperate and subtropical zone of the Northern Hemisphere, from North America through Europe and North Africa to China, Japan and the Philippines (4).

Nearly all Rosa spp. are characterized by: 1 . odd pinnate leaves, as only few species have single leaves; 2 . stems with prickles; 3 . cup-shaped flowers with the corolla composed of usually 5 , rarely 4 petals; 4 . in the centre of the flower, numerous styles surrounded by a hypanthium and a circle of stamens; 5 . the aggregate fruit, called a rose hip, with an outer fleshy layer and containing numerous achenes, which are the true rose fruits.

The attractiveness of roses is determined not only by the beautiful, fragrant flowers. Their additional ornamental features are fruits, interesting prickles, plant habit, stem colour, and leaf discoloration in autumn. Although wild roses have only single flowers, garden roses are characterized by a broad spectrum of flower forms: 1 . single flowers composed of 5 (rarely 4 ) petals and a normal number of stamens and pistils, which can produce fruits; 2 . semi-double flowers, with 10-20 petals, but also well-developed stamens and pistils, which also can produce fruits; 3 . double flowers with usually 20-40 petals (sometimes even over 100), but no fertile stamens and pistils, so they cannot produce fruits. Additional petals are modified stamens or styles -4 .

Roses have prickles and small thorns. Hardly anybody selects roses in respect of their armament. The diversity of forms of prickles and thorns is fascinating and additionally enhances the plant's ornamental value. They can be hair-like setae or strong, very broad, winged prickles (4).

Roses produce rose hips, which not only ornament them in autumn but also attract various birds. Thanks to the varied forms of these aggregate fruits (ranging from flattened to narrowly elliptic), their size and colours (red, orange, black), they can enrich the appearance of the garden (4).

As early as 5,000 years ago, rose was bred in Sumer, from where it spread to the whole Mesopotamia. At that time (2,600 BC), roses were also cultivated in China. Herodotus (490 BC) mentions King Midas, who took with him Phrygian roses when exiled to Macedonia. Apparently, some of the roses were double-flowered, with up to 60 petals. The oldest illustration of roses was found in the palace in Knossos on Crete (about 1,500 BC) but it is unclear if it was a garden rose or a wild rose. Only at the time when Persia flourished, roses became the most valuable garden plants. From Persia the rose started its journey to the European horticulture: to ancient Greece and Rome. The ancient 
Romans also grew roses in gardens and already knew many methods of their propagation, even by shield budding. Roses gained importance not only in gardens but also in everyday life and politics. During holidays and public speeches, roses became indispensable items. In the Middle Ages, initially their cultivation was stopped, as roses were associated with pagans. However, Charlemagne issued a decree that in each garden at least one rose had to be grown. Rose cultivation developed intensively in the 19th century. Till the 17th century only so-called old roses were known, and in the early 19th century the era of modern rose started, with diverse flower colours and forms, known to this day. At that time, Chinese rose (Rosa chinensis) was popularized, after its introduction to Europe probably in 1781. Many cultivars derive from this species, e.g. the tea roses with strongly fragrant, double flowers. Also in Poland, rose breeding has a long tradition and many achievements. The first exhibition of roses and other flowers in our country took place in 1882 in Warsaw $(4,5)$.

The symbolic meanings and legends linked with roses are very rich. Already in Antiquity, roses had a place in religion, art, and literature. The Greek poet Sappho in the 6th century BC was the first one to call the rose "queen of all flowers" (5). Romans celebrated a festival of roses (Rosalia), when they placed the flowers at burial sites. The wild rose is a symbol of mortality: its flower heralds death, and a broken rose indicates a suddenly or prematurely interrupted life (9).

The development of Christianity has brought new cultural and symbolic meanings of plant ornaments used in cemeteries and home gardens. Many of the plants traditionally used in cemeteries have a symbolic meaning, linked with Christian faith. The most conspicuous among them is rose. In Christian culture, roses are symbols for Mary and Christ's blood, given many religious meanings. White roses are a symbol of virginity, the love of Mother of God to her Son, and resurrection. Red roses symbolize martyrdom or prayer and mortification, especially of Jesus Christ, because of the blood colour and pentamerous perianth (in relation to the 5 wounds). Rose prickles denote suffering, sin, and the crown of thorns. Withering white roses, like no others apparently, draw attention to the fragility of life and passing (19).

\section{MATERIALS AND METHODS}

When mapping the distribution of Rosa spp. in the study area, the ATPOL cartogram method was used, according to the assumptions of the "Atlas of distribution of vascular plants in Poland" (13). The list of localities includes symbols of squares $(10 \mathrm{~km} \times 10 \mathrm{~km})$ on the ATPOL grid of Poland. The limits of Wielkopolska were delineated according to Żukowski et al. (18).

Many of the localities included in this list are recorded on the basis of herbarium specimens collected by the author in the field, but identified in the laboratory by the eminent specialist Prof. Dr hab. Jerzy Zieliński (Institute of Dendrology, Polish Academy of Sciences, Kórnik). The only exceptions are Rosa canina, $R$. jundzilli, R. multiflora, $R$. villosa, and $R$. zalana, which were identified only in the field.

A collection of Rosa spp. was established in the Dendrological Garden in Poznań (Department of Botany, Poznań University of Life Sciences). It was created because of difficulties with their identification in the field: a lack of flowers or hypanthium, very shaded conditions in some cemeteries, and frequent hybrids, either spontaneous or cultivated. Aboveground shoots with underground parts were transferred to the garden. Currently the collection includes 84 specimens of roses. They were identified by Prof. Dr hab. Jerzy Zieliński.

\section{LISTS OF TAXA AND THEIR LOCALITIES}

The taxa are listed in alphabetical order within 2 groups: native and alien taxa of the genus Rosa. For each taxon, localities are listed according to the order of ATPOL squares. After the name 
of the village/town/city, the name of province (voivodship) is given. Abbreviations and symbols: $\mathrm{B}$ - shrub layer; $\mathrm{C}$ - herb layer; $\mathrm{r},+, 1,2,3,4,5$ - cover-abundance according to the Braun-Blanquet scale (if the number was followed by a dot and 1, it denoted low sociability, i.e. the plants did not form clumps); leg. - collected by; det. - identified by.

\section{LIST OF NATIVE TAXA OF THE GENUS ROSA}

\section{Rosa canina L. (leg. det. A. Czarna)}

AC53 Kostrzyn on the Oder, Lubuskie: B1.1 (04.03.2015); AC59 Gościmiec near Zwierzyn, Lubuskie: B+ (07.05.2013); AC59 Górki Noteckie near Zwierzyn, Lubuskie: B1.1 (08.03.2015); AC59 Łącznica near Stare Kurowo, Lubuskie: B+ (08.03.2015); AC59 Zagaje near Zwierzyn, Lubuskie: B+ (07.05.2013); AC67 Osiedle Poznańskie near Deszczno, Lubuskie: B+ (21.09.2006); AC68 Gralewo near Santok, Lubuskie: B+ (11.11.2011); AC68 Nowe Polichno near Santok, Lubuskie: B+ (11.08.2006); AC68 Santok, Lubuskie: B+ (07.05.2015); AC68 Stare Polichno near Santok, Lubuskie: B+ (15.04.2010); AC69 Górecko near Zwierzyn, Lubuskie: B+ (10.11.2011); AC69 Jastrzębnik near Santok, Lubuskie: B+ (15.04.2010); AC69 Ludzisławice near Santok, Lubuskie: B+ (11.08.2006); AC73 Dobroszyn near Witnica, Lubuskie: B1.1 (04.03.2015); AC74 Kłopotowo near Witnica, Lubuskie: B+ (10.09.2006); AC75 Białcz near Witnica, Lubuskie B1.1 (05.03.2015); AC75 Białczyk near Witnica, Lubuskie: B+ (27.10.2011); AC75 Krępiny near Krzeszyce, Lubuskie: B+ (10.09.2006); AC75 Oksza near Witnica, Lubuskie: B+ (10.09.2006); AC75 Przemysław near Krzeszyce, Lubuskie: B+ (29.05.2010); AC75 Świerkocin near Witnica, Lubuskie: B+ (27.10.2011); AC75 Zaszczytowo near Krzeszyce, Lubuskie: B+ (10.09.2006); AC76 Bogdaniec, Lubuskie: B+ (27.10.2011); AC776 Glinik near Deszczno, Lubuskie: B+ (11.05.2010); AC76 Jasieniec near Bogdaniec, Lubuskie: B+ (28.10.20110; AC76 Jasieniec near Bogdaniec, Lubuskie: B+ (06.03.2015); AC76 Jeżyki near Bogdaniec, Lubuskie: B2.2 (06.03.2015); AC77 Łagodzin near Deszczno, Lubuskie: B+ (21.09.2006); AC77 Prądocin near Deszczno, Lubuskie: B+ (21.09.2006); AC79 Świniary Kol. near Skwierzyna, Lubuskie: B+ (28.04.2010); AC84 Czarnów near Górzyca, Lubuskie: B+ (30.05.2010); AC84 Ownice near Słońsk, Lubuskie: B+ (20.09.2006); AC84 Słońsk, Lubuskie: B+ (13.09.2006); AC85 Głuchów near Słońsk, Lubuskie: B+ (29.05.2010); AC85 Lemierzyce near Słońsk, Lubuskie: B+ (10.09.2006); AC85 Rudnica near Krzeszyce, Lubuskie: B+ (20.09.2006); AC86 Maszków near Krzeszyce, Lubuskie: B+ (12.05.2010); AC88 Bledzew, Lubuskie: B+ (22.09.2006); AC89 Twierdzielewo near Przytoczna, Lubuskie: B+ (08.09.2006); AC90 Bobowicko near Międzyrzecz, Lubuskie: B+Cr (04.05.2010); AC93 Golice near Stubice, Lubuskie: B+ (31.05.2010); AC93 Laski Lubuskie near Górzyca, Lubuskie: B+ (30.05.2010); AC93 Owczary near Górzyca, Lubuskie: B+ (30.05.2010); AC93 Pławidło near Słubice, Lubuskie: B+ (31.05.2010); AC94 Ośno Lubuskie, Lubuskie: B+ (12.09.2006); AC95 Radachów near Ośno Lubuskie, Lubuskie: B+ (19.06.2006); AC95 Trześniów near Ośno Lubuskie, Lubuskie: B+ (27.05.2010); AC96 Miechów near Sulęcin, Lubuskie: B+ (10.09.2006); AC97 Trzemeszno Lubuskie near Sulęcin, Lubuskie: B+ (21.09.2006); AC99 Kalsko near Międzyrzecz, Lubuskie: B+ (05.05.2010); AC99 Międzyrzecz, Lubuskie: B+ (05.05.2010); AD02 Nowy Lubusz near Słubice, Lubuskie: B+ (13.09.2006); AD03 Lisów near Słubice, Lubuskie: B+ (31.05.2010); AD03 Starków near Rzepin, Lubuskie: B+ (12.09.2006); AD04 Rzepin, Lubuskie: B+ (14.09.2006); AD08 Wielowieś near Sulęcin, Lubuskie: B+ (22.09.2006); AD09 Kęszyca near Międzyrzecz, Lubuskie: B+ (06.05.2010); AD09 Nietoperek near Trzciel, Lubuskie: B+ (07.09.2006); AD13 Kunice near Słubice, Lubuskie: B+ (06.85.2008); AD13 Maczków near Cybinka, Lubuskie: B+ (26.09.2006); AD18 Bucze near Lubrza, Lubuskie: B+ (07.05.2010); AD18 Lubrzạ, Lubuskie: B+ (18.05.2010); AD18 Wilkowo near Świebodzin, Lubuskie: B+ (17.04.2007); AD18 Zagaje near Lubrza, Lubuskie: B+ (22.09.2006); AD19 Rzeczyca near Świebodzin, Lubuskie: B+, Cr (30.06.2011); AD19 Wolimirzyce near Szczaniec, Lubuskie: B+ (16.04.2007); AD24 Cybinka, Lubuskie: B+ (26.09.2006); 
AD24 Drzeniów near Cybinka, Lubuskie: B+ (25.09.2006); AD24 Sądów near Cybinka, Lubuskie: B+ (26.09.2006); AD25 Bargów near Torzym, Lubuskie: B+ (26.09.2006); AD26 Dobrosułów near Bytnica, Lubuskie: B+ (12.06.2010); AD27 Gryżyna near Bytnica, Lubuskie: B+ (23.09.2006); AD27 Mostki near Lubrza, Lubuskie: B+ (09.05.2010); AD28 Lubrza, Lubuskie: B+ (23.09.2006); AD29 Jeziory near Świebodzin, Lubuskie: B+ (16.04.2007); AD29 Rzeczyca near Świebodzin, Lubuskie: B+ (17.04.2007); AD34 Bytomiec near Maszewo, Lubuskie: B+ (25.09.2006); AD34 Rybaki near Maszewo, Lubuskie: B+ (25.09.2006); AD35 Granice near Maszewo, Lubuskie: B+ (04.06.2010); AD35 Skarbona near Maszewo, Lubuskie: B+ (25.09.2006); AD36 Bytnica, Lubuskie: B+ (24.09.2006); AD36 Łąkie near Skąpe, Lubuskie: B+ (09.05.2010); AD38 Międzylesie near Skąpe, Lubuskie: B+ (09.05.2010); AD39 Kalsk near Sulechów, Lubuskie: B+ (29.09.2011); AD39 Kije near Sulechów, Lubuskie: B+ (01.05.2007); AD44 Chlebowo near Gubin, Lubuskie: B+ (13.06.2010); AD44 Drzeńsk Wielki near Gubin, Lubuskie: B+, Cr (14.06.2010); AD44 Połęcko near Maszewo, Lubuskie: B+ (25.09.2006); AD45 Chojna near Krosno Odrzańskie, Lubuskie: B+ (13.06.2010); AD45 Wężyska near Krosno Odrzańskie, Lubuskie: B+ (13.06.2010); AD46 Dąbie, Lubuskie: B+ (10.06.2010); AD46 Nowy Zagór near Dąbie, Lubuskie: B+ (10.06.2010); AD46 Stary Radruszec near Krosno Odrzańskie, Lubuskie: B+ (13.06.2010); AD46 Stary Zagór, near Dąbie, Lubuskie: B+ (21.09.2008); AD47 Szczawno near Dąbie, Lubuskie: B+ (10.06.2010); AD48 Wysokie near Czerwieńsk, Lubuskie: B+ (09.06.2010); AD49 Krępa near Zielona Góra, Lubuskie: B+ (25.08.2008); AD53 Bieżyce near Gubin, Lubuskie: B+ (18.09.2008); AD53 Gubin, Lubuskie: B+ (22.03.2014); AD53 Gubinek near Gubin, Lubuskie: B+ (21.08.2014); AD54 Czeklin near Bobrowice, Lubuskie: B+ (19.09.2008); AD54 Kaniów near Gubin, Lubuskie: B+ (19.09.2008); AD54 Pole, near Gubin, Lubuskie: B+ (19.09.2008); AD54 Przyborowice near Gubin, Lubuskie: B+ (18.09.2008); AD55 Przychów near Bobrowice, Lubuskie: B+ (21.09.2008); AD55 Wełmice near Bobrowice, Lubuskie: B1.1 (26.03.2014); AD56 Bobrowice, Lubuskie: B+ (03.04.2014); AD56 Dęby near Bobrowice, Lubuskie: B1.1 (03.04.2014); AD57 Drzonów near Świdnica, Lubuskie: B+ (02.04.2014); AD57 Letnica near Świdnica, Lubuskie: B+ (02.04.2014); AD57 Lipno near Świdnica, Lubuskie: B+, Cr (27.08.2008); AD58 Świdnica, Lubuskie: B+ (27.08.2008); AD63 Brody, Lubuskie: B+ (28.03.2014); AD63 Jasienica near Brody, Lubuskie: B+ (24.03.2014); AD63 Koło near Brody, Lubuskie: B+ (20.09.2008); AD63 Sadzarzewice near Gubin, Lubuskie: B+ (20.09.2008); AD63 Stargard Gubiński near Gubin, Lubuskie: B+ (18.09.2008); AD63 Węgliny near Gubin, Lubuskie: B+ (20.09.2008); AD63 Wielotów near Gubin, Lubuskie: B+ (20.09.2008); AD63 Witaszkowo near Gubin, Lubuskie: B+ (19.09.2008); AD64 Jałowice near Brody, Lubuskie: B+ (18.09.2008); AD64 Laski near Brody, Lubuskie: B+ (18.09.2008); AD64 Osiek near Lubsko, Lubuskie: B+ (15.09.2008); AD64 Raszyn near Lubsko, Lubuskie: B1.1 (7.03.2014); AD64 Starosiedle near Gubin, Lubuskie: B+ (18.09.2008); AD65 Chocicz near Lubsko, Lubuskie: B+, Cr (21.09.2008); AD65 Dłużek near Lubsko, Lubuskie: B1.1 (15.09.2008); AD65 Lubsko, Lubuskie: B1.1 (15.09.2008); AD65 Tymienice near Lubsko, Lubuskie: B+ (15.09.2008); AD66 Łagoda near Nowogród Bobrzański, Lubuskie: B+ (03.04.2014); AD67 Kaczeniec near Nowogród Bobrzański, Lubuskie: B+ (29.08.2008); AD67 Koźla near Świdnica, Lubuskie: B+ (27.08.2008); AD67 Podgórzyce near Nowogród Bobrzański, Lubuskie: B+ (31.08.2008); AD67 Sobolice near Nowogród Bobrzański, Lubuskie: B+ (29.08.2008); AD67 Sterków near Nowogród Bobrzański, Lubuskie: B+ (02.04.2014); AD69 Czasław near Otyń, Lubuskie: B+ (26.08.2008); AD69 Książ Śląski near Kożuchów, Lubuskie: B+ (26.08.2008); AD69 Lubieszów near Nowa Sól, Lubuskie: B+ (26.08.2008); AD69 Ługi near Otyń, Lubuskie: B+ (26.08.2008); AD74 Grabów near Tuplice, Lubuskie: B+ (30.03.2014); AD74 Jasionna near Jasień, Lubuskie: B+ (16.09.2008); AD74 Jurzyn near Jasień, Lubuskie: B+ (01.04.2014); AD74 Tuplice, Lubuskie: B+ (31.03.2014); AD76 Kadłubia near Jasień, Lubuskie: B+ (22.09.2009); AD77 Dobroszów Wielki near Nowogród Bobrzański, Lubuskie: B+, Cr (18.08.2009); AD77 Dybów near Żagań, Lubuskie: B+ (14.08.2009); AD77 Gorzupia near Żary, Lubuskie: B+ (14.09.2008); AD77 Przybymierz near Nowogród Bobrzański, Lubus- 
kie: B+ (18.08.2009); AD77 Stanów near Brzeźnica, Lubuskie: B+ (14.09.2008); AD78 Broniszów near Kożuchów, Lubuskie: + (17.08.2009); AD78 Chotków near Brzeźnica, Lubuskie: B+ (14.09.2008); AD78 Marcinów near Brzeźnica, Lubuskie: B+ (18.08.2009); AD78 Skibice near Nowogród Bobrzański, Lubuskie: B+ (18.08.2009); AD78 Wichów near Brzeźnica, Lubuskie: B+ (17.08.2009); AD79 Kożuchów, Lubuskie: B+ (28.08.2008); AD79 Leśniów Wlk. near Czerwieńsk, Lubuskie: B+ (27.08.2008); AD79 Stypułów near Kożuchów, Lubuskie: B+ (14.09.2008); AD85 Górka near Lipinki Łużyckie, Lubuskie: B+ (20.05.2009); AD86 Żary, Lubuskie: B+ (24.09.2009); AD87 Olszyniec near Żary, Lubuskie: B+ (04.04.2014); AD87 Żagań, Lubuskie: B+ (14.04.2009); AD88 Karczówka near Brzeźnica, Lubuskie: B+, Cr (09.04.2012); AD89 Borów near Nowe Miasteczko, Lubuskie: B+ (09.09.2008); AD94 Chudzowice near Trzebiel, Lubuskie: B+ (16.08.2009); AD94 Dębinka near Trzebiel, Lubuskie: B+ (16.08.2009); AD94 Jagłowice near Trzebiel, Lubuskie: B+ (16.08.2009); AC96 Sulęcin, Lubuskie: B+ (11.09.2006); AC97 Lubniewice, Lubuskie: B+ (21.09.2006); BC17 Dolaszewo near Szydłowo, Wielkopolskie: B+ (06.08.2011); BC17 Piła, Wielkopolskie: B+ (29.04.2014); BC18 Brodna near Kaczory, Wielkopolskie: B+ (26.08.2010); BC26 Kępa near Trzcianka, Wielkopolskie: B+ (02.09.2011); BC26 Kłoda near Szydłowo, Wielkopolskie: B+ (10.03.2015); BC26 Łomnica-Młyn near Trzcianka, Wielkopolskie: B+ (12.03.2015); BC27 Byszki near Ujście, Wielkopolskie: B+ (23.08.2010); BC27 Dolaszewo near Szydłowo, Wielkopolskie: B+ (13.03.2015); BC27 Kotuń near Szydłowo, Wielkopolskie: B+ (10.03.2015); BC27 Ługi Ujskie near Ujście, Wielkopolskie: B+ (07.09.2011); BC27 Piła, Wielkopolskie: B+ (29.04.2014); BC28 Piła, Wielkopolskie: B+ (29.04.2014); BC28 Równopole near Kaczory, Wielkopolskie: B+, Cr (25.08.2010); BC29 Miasteczko Krajeńskie, Miasteczko Krajeńskie, Wielkopolskie: B+ (24.09.2011); BC35 Siedlisko near Trzcianka, Wielkopolskie: B+ (11.05.2014); BC36 Biała near Trzcianka, Wielkopolskie: B1.1 (12.05.2014); BC35 Trzcianka, Wielkopolskie: B+ (12.03.2015); BC36 Radolin near Trzcianka, Wielkopolskie: B+ (12.05.2014); BC36 Radolinek near Czarnków, Wielkopolskie: B+ (10.05.2014); BC36 Romanowo Górne near Czarnków, Wielkopolskie: B1.1 (16.07.2006); BC37 Nowa Wieś Ujska near Ujście, Wielkopolskie: B+ (01.05.2014); BC37 Ujście, Wielkopolskie: B1.1 (01.05.2014); BC37 Węglewo near Ujście, Wielkopolskie: B+ (08.05.2014); BC38 Chodzież, Wielkopolskie: B+ (15.07.2006); BC38 Chrustowo near Ujście, Wielkopolskie: B1.1 (01.05.2014); BC38 Cisze near Chodzież, Wielkopolskie: B+ (21.05.2006); BC38 Studzieniec near Chodzież, Wielkopolskie: B+ (01.05.2014); BC39 Nałęcza near Szamocin, Wielkopolskie: B+ (18.03.2015); BC39 Strzelce near Chodzież, Wielkopolskie: B+ (19.09.2005); BC42 Brzegi near Krzyż Wlkp., Wielkopolskie: B+ (05.09.2011); BC42 Drawiny near Drezdenko, Lubuskie: B+ (08.05.2013); BC42 Łokacz Wielki near Krzyż Wlkp., Wielkopolskie: B+ (08.05.2013); BC43 Herbutowo near Wieleń, Wielkopolskie: B1.1 (09.03.2015); BC43 Lubcz Mały near Krzyż Wlkp., Wielkopolskie: B+ (05.09.2011); BC43 Wieleń, Wielkopolskie: B+, Cr (24.07.2006); BC44 Folsztyn near Wieleń, Wielkopolskie: B+ (10.05.2014); BC44 Nowe Dwory near Wieleń, Wielkopolskie: B1.1 (10.05.2014); BC44 Zielonowo near Wieleń, Wielkopolskie: B+ (12.05.2014); BC45 Gajewo near Czarnków, Wielkopolskie: B+ (10.05.2014); BC45 Jędrzejewo near Czarnków, Wielkopolskie: B+ (10.05.2014); BC45 Radosiew near Czarnków, Wielkopolskie: B+ (04.09.2011); BC45 Runowo near Trzcianka, Wielkopolskie: B+ (21.05.2014); BC46 Brzeźno near Czarnków, Wielkopolskie: B+ (09.05.2014); BC46 Kuźnica Czarnkowska near Czarnków, Wielkopolskie: B+ (12.05.2014); BC46 Romanowo Dolne near Czarnków, Wielkopolskie: B+ (08.05.2014); BC46 Zofiewo near Czarnków, Wielkopolskie: B+ (09.05.2014); BC47 Paliszewo near Czarnków, Wielkopolskie: B+ (16.07.2006); BC48 Ostrówki near Budzyń, Wielkopolskie: B+ (02.05.2014); BC48 Stróżewice near Chodzież, Wielkopolskie: B+ (02.05.2014); BC49 Adolfowo near Margonin, Wielkopolskie: B+ (02.05.2014); BC49 Budzyń, Wielkopolskie: B1.1 (18.09.2005); BC49 Podstolice near Budzyń, Wielkopolskie: B+ (19.05.2006); BC49 Radwanki near Margonin, Wielkopolskie: B+ (02.05.2014); BC50 Błotno near Zwierzyn, Lubuskie: B+ (07.05.2013); BC50 Pławin near Stare Kurowo, Lubuskie: B+ (12.11.2011); BC50 Przynotecko 
near Stare Kurowo, Lubuskie: B+ (10.11.2011); BC50 Trzebicz near Drezdenko, Lubuskie: B+ (06.05.2013); BC50 Żółwin near Zwierzyn, Lubuskie: B+ (07.05.2013); BC51 Drezdenko, Lubuskie: B+ (08.05.2013); BC51 Kosin near Drezdenko, Lubuskie: B+ (11.05.2010); BC51 LipnoTuczępy near Drezdenko, Lubuskie: B+ (10.08.2006); BC51 Marzenin near Drezdenko, Lubuskie: B+ (10.04.2014); BC51 Pełcza near Drezdenko Drawsko, Wielkopolskie: B+ (10.08.2006); BC51 Stare Bielice near Drezdenko, Lubuskie: B+ (08.03.2015); BC52 Chełst near Krzyż Wlkp. Drawsko, Wielkopolskie: B+ (10.08.2006); BC52 Kamiennik near Krzyż Wlkp. Drawsko, Wielkopolskie: B+ (07.04.2010); BC52 Nowe Bielice near Krzyż Wlkp., Wielkopolskie: B+ (07.09.2011); BC54 Rosko near Wieleń, Wielkopolskie: B+ (07.04.2010); BC54 Wrzeszczyna near Wieleń, Wielkopolskie: B+ (07.04.2010); BC55 Kruteczek near Lubasz, Wielkopolskie: B+ (07.04.2010); BC56 Białężyn near Czarnków, Wielkopolskie: B+ (17.07.2006); BC56 Jędrzejewo near Lubasz, Wielkopolskie: B1.1 (10.04.2010); BC56 Lubasz, Wielkopolskie: B+ (07.04.2010); BC58 Bukowiec near Budzyń, Wielkopolskie: B+ (16.09.2011); BC58 Nowa Wieś Wyszyńska near Budzyń., Wielkopolskie: B+ (25.04.2006); BC58 Ryczywół, Wielkopolskie: B+ (03.05.2014); BC58 Tłukawy near Ryczywół, Wielkopolskie: B+ (25.09.2011); BC58 Wyszynki near Budzyń, Wielkopolskie: B+ (25.04.2006); BC58 Zawady near Ryczywół, Wielkopolskie: B+ (25.09.2011); BC59 Brzekiniec near Wągrowiec, Wielkopolskie: B+, Cr (02.09.2005); BC59 Nowe Brzeźno near Budzyń, Wielkopolskie: B+ (02.09.2005); BC60 Goszczanowiec near Drezdenko, Lubuskie: B+ (27.04.2010); BC60 Gościm near Drezdenko, Lubuskie: B+ (15.04.2010); BC61 Grotów near Drezdenko, Lubuskie: B+ (07.04.2010); BC61 Lubiatów near Drezdenko, Lubuskie: B+ (26.04.2010); BC62 Kwiejce near Krzyż Wlkp., Wielkopolskie: B+ (07.04.2010); BC64 Chojno near Wronki, Wielkopolskie: B+ (25.07.2006); BC65 Jasionna near Wronki, Wielkopolskie: B+ (07.04.2010); BC68 Gościejewko near Ryczywół, Wielkopolskie: B+ (25.09.2011); BC68 Jaracz near Rogoźno Wlkp., Wielkopolskie: B+ (23.04.2006); BC68 Tarnowo near Rogoźno Wlkp., Wielkopolskie: B+ (23.04.2006); BC69 Potuły near Wągrowiec, Wielkopolskie: B1.1 (07.05.2014); BC69 Rogoźno Wlkp., Wielkopolskie: B+ (23.04.2014); BC70 Nowe Dłusko near Przytoczna, Lubuskie: B+ (01.05.2010); BC70 Wiejce near Skwierzyna, Wielkopolskie: B+ (14.08.2006); BC71 Mierzyn near Międzychód, Wielkopolskie: B+ (28.04.2010); BC71 Mokrzec near Międzychód, Wielkopolskie: B+ (28.04.2010); BC73 Kaczlin near Sieraków, Wielkopolskie: B+ (06.08.2006); BC73 Lutom near Sieraków, Wielkopolskie: B+ (04.08.2006); BC73 Ryżyn near Chrzypsko Wielkie, Wielkopolskie: B+ (06.08.2006); BC73 Tuchola near Sieraków, Wielkopolskie: B+ (04.05.2007); BC74 Białcz near Chrzypsko Wielkie, Wielkopolskie: B+ (06.08.2006); BC76 Jaryszewo near Obrzycko, Wielkopolskie: B+ (20.07.2006); BC76 Piotrkówki near Szamotuły, Wielkopolskie: B+ (08.05.2007); BC76 Szamotuły, Wielkopolskie: B+ (08.05.2007); BC77 Kiszewo near Oborniki, Wielkopolskie: B+ (05.04.2010); BC77 Nowołoskoniec near Oborniki, Wielkopolskie: B+ (06.04.2010); BC77 Słonawy near Oborniki, Wielkopolskie: B+ (06.04.2010); BC77 Uścikowo near Oborniki, Wielkopolskie: $\mathrm{B}+, \mathrm{Cr}$ (15.09.2007); BC78 Łukowo near Oborniki, Wielkopolskie: B+ (15.09.2007); BC78 Oborniki, Wielkopolskie: B+ (15.09.2007); BC79 Białęgi near Murowana Goślina, Wielkopolskie: B+ (21.04.2014); BC79 Budziszewko near Rogoźno Wlkp., Wielkopolskie: B+ (22.04.2014); BC79 Nienawiszcz near Rogoźno Wlkp., Wielkopolskie: B+ (22.04.2014); BC79 Słomowo near Rogoźno Wlkp., Wielkopolskie: B+ (22.04.2014); BC79 Stary Łoskoń near Murowana Goślina, Wielkopolskie: B+ (22.04.2014); BC79 Studzieniec near Rogoźno Wlkp., Wielkopolskie: B+ (22.04.2014); BC80 Zielomyśl near Pszczew, Lubuskie: B+ (16.08.2006); BC81 Nowe Gorzycko near Pszczew, Lubuskie: B+ (02.05.2010); BC81 Stare Gorzycko near Międzychód, Wielkopolskie: B+ (16.08.2006); BC82 Kolno near Międzychód, Wielkopolskie: B+ (10.04.2010); BC82 Mechnacz near Kwilcz, Wielkopolskie: B+ (07.08.2006); BC84 Nojewo near Pniewy, Wielkopolskie: B+ (07.05.2007); BC84 Psarki near Pniewy, Wielkopolskie: B+ (08.08.2006); BC84 Rudki near Pniewy, Wielkopolskie: B+ (08.08.2006); BC85 Buszewo near Pniewy, Wielkopolskie: B+ (02.09.2006); BC85 Zajączkowo near Pniewy, Wielkopolskie: B+ (20.04.2010); BC86 Sokółki Małe near 
Kazimierz, Wielkopolskie: B+ (07.05.2007); BC87 Cerekwica near Rakoniewice, Wielkopolskie: B+ (08.05.2007); BC89 Murowana Goślina, Wielkopolskie: B+ (21.04.2014); BC89 Złotoryjsko near Murowana Goślina, Wielkopolskie: B+ (16.09.2007); BC91 Łowyń near Międzychód, Wielkopolskie: B+ (02.05.2010); BC91 Stoki near Pszczew, Lubuskie: B+ (02.05.2010); BC92 Błaki near Miedzichowo, Wielkopolskie: B+, Cr (04.09.2006); BC93 Komorowice near Lwówek, Wielkopolskie: B+ (04.09.2006); BC95 Niewierz near Duszniki, Wielkopolskie: B+ (02.09.2006); BC97 Kobylniki Małe near Rokietnica, Wielkopolskie: B+ (03.06.2007); BC98 Poznań-Morasko near Poznań, Wielkopolskie: B+ (03.06.2014); BC98 Złotkowo near Suchy Las, Wielkopolskie: B+ (21.04.2014); BC97 Kobylniki Małe near Rokietnica, Wielkopolskie: B+ (03.06.2007); BC99 Owińska near Czerwonak, Wielkopolskie: B+ (04.05.2014); BD00 Siecz near Trzciel, Lubuskie: B+ (07.09.2006); BD01 Trzciel, Lubuskie: B+ (21.04.2010); BD02 Łęczno near Miedzichowo, Wielkopolskie: B+ (05.09.2006); BD03 Mała Lipka near Nowy Tomyśl, Wielkopolskie: B+ (05.09.2006); BD04 Chraplewo near Kuślin, Wielkopolskie: B+ (26.05.2010); BD04 Głuponia near Kuślin, Wielkopolskie: B+ (26.05.2010); BD04 Kuślin, Wielkopolskie: B+ (03.09.2006); BD04 Władysławowo near Lwówek, Wielkopolskie: B+ (03.09.2006); BD05 Sędziny near Duszniki, Wielkopolskie: B+ (02.09.2006); BD05 Śliwno near Kuślin, Wielkopolskie: B+ (26.05.2010); BD06 Buk, Wielkopolskie: B+ (1.08.2006); BD06 Buk, Wielkopolskie: B+ (31.08.2006); BD06 Wierzeja near Duszniki, Wielkopolskie: B+ (05.09.2006); BD07 Zakrzewo near Dopiewo, Wielkopolskie: B+ (01.06.2007); BD08 Żarzyn near Sulęcin, Lubuskie: B+ (10.05.2010); BD10 Brójce near Trzciel, Lubuskie: B+ (17.04.2007); BD10 Lutol Suchy near Trzciel, Lubuskie: B+ (01.07.2011); BD10 Rogoziniec near Zbąszynek, Lubuskie: B+ (17.04.2007); BD13 Chojniki near Nowy Tomyśl, Wielkopolskie: B+ (02.05.2005); BD13 Grubsko near Nowy Tomyśl, Wielkopolskie: B+ (02.05.2005); BD14 Kąkolewo near Grodzisk Wlkp., Wielkopolskie: B+, C+ (01.05.2005); BD20 Brudzewo near Szczaniec, Lubuskie: B+ (16.04.2007); BD20 Koźminek near Szczaniec, Lubuskie: B+ (01.07.2011); BD20 Przygubiel near Sulechów, Lubuskie: B+ (17.04.2007); BD22 Stefanów near Zbąszyń, Wielkopolskie: B+ (02.05.2007); BD23 Jabłonna near Rakoniewice, Wielkopolskie: B+ (13.04.2005); BD24 Elżbieciny near Rakoniewice, Wielkopolskie: B+ (17.04.2005); BD29 Radzewice near Mosina, Wielkopolskie: B+ (12.06.2005); BD31 Kopanica near Siedlec, Wielkopolskie: B+ (18.04.2007); BD33 Głodno near Rakoniewice, Wielkopolskie: B+ (17.04.2005); BD33 Rostarzewo near Rakoniewice, Wielkopolskie: B+ (17.04.2005); BD33 Stary Widzim near Wolsztyn, Wielkopolskie: B+ (19.04.2005); BD35 Kotusz near Kamieniec, Wielkopolskie: B+ (13.11.2011); BD36 Kiełczewo near Kościan, Wielkopolskie: B+ (08.11.2012); BD37 Czempiń, Wielkopolskie: B+ (26.04.2005); BD39 Dolsk, Wielkopolskie: B1.1 (14.06.2005); BD40 Klenica near Bojadła, Lubuskie: B+ (13.09.2008); BD40 Swarzynice near Trzebiechów, Lubuskie: B+ (30.04.2007); BD40 Trzebiechów, Lubuskie: B+ (30.04.2007); BD41 Bełcze near Bojadła, Lubuskie: B+ (13.09.2008); BD42 Nowy Jaromierz near Kargowa, Lubuskie: B+ (29.04.2007); BD43 Nowa Wieś near Przemęt, Wielkopolskie: B+ (07.05.2012); BD50 Czarna near Zabór, Lubuskie: B1.1 (28.03.2012); BD50 Łazy near Zabór, Lubuskie: B+ (28.03.2012); BD50 Milsko near Zabór, Lubuskie: B+ (13.09.2008); BD51 Pyrnik near Bojadła, Lubuskie: B+ (13.09.2008); BD53 Śmieszkowo near Sława, Lubuskie: B+ (18.10.2011); BD56 Goniembice near Lipno, Wielkopolskie: B+ (20.06.2006); BD56 Żakowo near Lipno, Wielkopolskie: B+ (0.06.2006); BD59 Przytok near Zabór, Lubuskie: B+ (14.04.2012); BD60 Stany near Nowa Sól, Lubuskie: B+ (12.09.2008); BD62 Krążkowo near Sława, Lubuskie: B+ (20.04.2012); BD66 Popowo Wonieskie near Osieczna, Wielkopolskie: B+ (25.10.2011); BD67 Rydzyna, Wielkopolskie: B+ (04.06.2005); BD67 Tworzanice near Rydzyna, Wielkopolskie: B+ (04.06.2005); BD70 Kiełcz near Nowa Sól, Lubuskie: B+ (09.09.2008); BD70 Królikowice near Bytom Odrzański, Lubuskie: B+ (09.09.2008); BD70 Nowe Żabno near Nowa Sól, Lubuskie: B+ (09.09.2008); BD70 Sokołów near Kożuchów, Lubuskie: B+, Cr (02.04.2012); BD71 Siedlisko, Lubuskie: B+ (10.09.2008); BD71 Wierzbnice near Bytom Odrzański, Lubuskie: B+ (7.04.2012); BD73 Krzepielów near Sława, Lubuskie: B+ (07.07.2005); 
BD75 Brzeżany near Góra Śląska, Dolnośląskie: B+ (05.07.2005); BD75 Chróścina near Góra Śl., Dolnośląskie: B+ (12.05.2012); BD75 Łękanów near Niechlów, Dolnośląskie: B+ (06.07.2005); BD76 Czernina near Góra, Dolnośląskie: B2.2 (04.06.2005); BD76 Sułów near Góra Śląska, Dolnośląskie: B+ (04.07.2005); BD77 Kłoda near Rydzyna, Wielkopolskie: B+ (17.05.2012); BD78 Ziemlin near Krobia, Wielkopolskie: B+ (03.06.2005); BD80 Mycielin, near Niegosławice, Lubuskie: B+ (08.04.2014); BD80 Stara Jabłona near Niegosławice, Lubuskie: B+ (01.05.2014); BD81 Gaworzyce, Dolnośląskie: B+ (29.08.2009); BD81 Gostyń near Gaworzyce, Dolnośląskie: B+ (29.08.2009); BD81 Korytów near Gaworzyce, Dolnośląskie: B+ (29.08.2009); BD81 Koźlice near Gaworzyce, Dolnośląskie: B+ (29.08.2009); BD81 Nowa Jabłona near Niegosławice, Lubuskie: B+ (22.08.2009); BD81 Śrem near Gaworzyce, Dolnośląskie: B+ (28.04.2012); BD82 Biechów near Głogów, Dolnośląskie: B+ (28.08.2009); BD82 Dankowice near Żukowice, Dolnośląskie: B+ (29.08.2009); BD82 Drożów near Radwanice, Dolnośląskie: B+, Cr (29.08.2009); BD82 Kurowice near Jerzmanowa, Dolnośląskie: B1.1 (28.08.2009); BD82 Nielubia near Żukowice, Dolnośląskie: B+ (29.08.2009); BD82 Zabłocie near Żukowice, Dolnośląskie: B+ (29.08.2009); BD82 Żukowice, Dolnośląskie: B+ (10.09.2008); BD83 Bucze near Grębocice, Dolnośląskie: B+ (30.08.2009); BD83 Turów near Głogów, Dolnośląskie: B+ (31.08.2009); BD84 Bełcz Wlk. near Niechlów, Dolnośląskie B+ (02.09.2009); BD84 Głobice near Niechlów, Dolnośląskie: B+ (02.09.2009); BD84 Gołkowice near Pęcław, Dolnośląskie: B+ (25.04.2012); BD84 Kotowice near Pęcław, Dolnośląskie: B+ (30.08.2009); BD84 Wągroda near Niechlów, Dolnośląskie: B+ (02.09.2009); BD85 Naratów near Niechlów, Dolnośląskie: B+ (13.05.2012); BD85 Rogów Górowski near Góra Śląska, Dolnośląskie: B+ (02.07.2005); BD85 Strumienna near Góra Śląska, Dolnośląskie: B+ (05.07.2005); BD85 Szaszorowice near Niechlów, Dolnośląskie: B+ (04.07.2005); BD85 Wroniniec near Niechlów, Dolnośląskie: B+ (13.05.2012); BD86 Góra Śląska, Dolnośląskie: B+ (01.07.2005); BD92 Gaiki near Jerzmanowa, Dolnośląskie: B1.1 (28.08.2009); BD92 Jakubów near Radwanice, Dolnośląskie: B+ (28.08.2009); BD92 Kaźmierzów near Polkowice, Dolnośląskie: B+, Cr (27.04.2012); BD92 Moskorzyn near Polkowice, Dolnośląskie: B+ (26.08.2009); BD93 Duża Wólka near Grębocice, Dolnośląskie: B+ (31.08.2009); BD94 Kłębów near Rudna, Dolnośląskie: B+ (01.09.2009); BD95 Chobieniek near Rudna, Dolnośląskie: B+ (02.09.2009); BD95 Kurów Mały near Jerzmanowa, Dolnośląskie: B+ (31.08.2009); BD95 Kwielice near Grębocice, Dolnośląskie: B+ (31.08.2009); BD96 Psary near Jemielno, Dolnośląskie: B+ (03.09.2009); BD97 Górka Wąsowska near Wąsosz, Dolnośląskie: B+ (01.07.2005); BD97 Ługi near Wąsosz, Dolnośląskie: B+ (22.05.2012); BD97 Płoski near Wąsosz, Dolnośląskie: B+ (03.09.2009); BD98 Radziądz near Żmigród, Dolnośląskie: B+ (25.07.2010); CC15 Kruszyniec near Siecinko, Kujawsko-Pomorskie: B1.1 (20.03.2015); CC20 Białośliwie, Wielkopolskie: B+ (07.09.2011); CC20 Helidorowo near Szamocin, Wielkopolskie: B+ (19.03.2015); CC20 Lipia Góra near Szamocin, Wielkopolskie: B+ (15.05.2006); CC20 Sokolec near Szamocin, Wielkopolskie: B+ (15.05.2006); CC21 Bąkowo Górne near Wyrzysk, Wielkopolskie: $\mathrm{B}+$ (09.09.2011); CC21 Osiek on the Noteć near Wyrzysk, Wielkopolskie: B+ (08.09.2011); CC22 Kowalewko near Kcynia, Kujawsko-Pomorskie: B+ (23.09.2011); CC24 Rynarzewo near Szubin, Kujawsko-Pomorskie: B1.1 (06.05.2006); CC24 Szkocja near Szubin, Kujawsko-Pomorskie: B+ (07.05.2006); CC25 Białe Błota, Kujawsko-Pomorskie: B+ (23.08.2008); CC25 Przyłęki near Białe Błota, Kujawsko-Pomorskie: B+ (22.08.2008); CC27 Wypaleniska near Solec Kujawski, Kujawsko-Pomorskie: B+, Cr (19.08.2008); CC30 Borowo near Szamocin, Wielkopolskie: B+ (14.05.2006); CC30 Borówki near Szamocin, Wielkopolskie: B+ (15.05.2006); CC30 Szamocin, Wielkopolskie: B+ (18.03.2015); CC31 Nowa Wieś Notecka near Kcynia, Kujawsko-Pomorskie: B1.1 (14.05.2006); CC32 Grocholin near Kcynia, Kujawsko-Pomorskie: B+ (17.05.2014); CC32 Iwno near Kcynia, Kujawsko-Pomorskie: B+ (17.05.2014); CC32 Kcynia, Kujawsko-Pomorskie B+ (03.09.2005); CC32 Miąskowo near Kcynia, Kujawsko-Pomorskie: B1.1 (17.05.2014); CC32 Stołężyn near Wapno, Wielkopolskie: B1.1 (17.05.2014); CC33 Chodzież, Wielkopolskie: B+ (19.09.2005); CC33 Słonawy near Szamocin, Wielkopolskie: B+ (10.05.2006); 
CC34 Kornelin near Szubin, Kujawsko-Pomorskie: B+ (07.05.2006); CC35 Małe Rudy near Szubin, Kujawsko-Pomorskie: B+ (06.05.2006); CC35 Władysławowo near Łabiszyn, Kujawsko-Pomorskie: B+ (06.05.2006); CC36 Bydgoszcz, Kujawsko-Pomorskie: B+ (20.08.2008); CC37 Solec Kujawski, Kujawsko-Pomorskie: B+(18.08.2008); CC40 Margonin, Wielkopolskie: B+(04.09.205); CC40 Margońska Wieś near Margonin, Wielkopolskie: B+ (04.09.205); CC40 Oporzyn near Wągrowiec, Wielkopolskie: B+ (02.09.2005); CC41 Gołańcz near Wągrowiec, Wielkopolskie: B+ (21.09.2013); CC41 Laskownica Wlk. near Gołańcz, Wielkopolskie: B+ (30.08.2005); CC41 Łekno near Wągrowiec, Wielkopolskie: B1.1 (26.04.2014); CC41 Oleszno near Gołańcz, Wielkopolskie: B+ (16.05.2014); CC43 Dziewierzewo near Kcynia, Kujawsko-Pomorskie B+ (17.05.2014); CC43 Królikowo near Szubin, Kujawsko-Pomorskie: B+ (22.09.2013); CC44 Brzyskorzystew near Żnin, Kujawsko-Pomorskie: B+ (22.09.2013); CC44 Dąbrówka Słupska near Szubin, Kujawsko-Pomorskie: B+ (22.09.2013); CC44 Wawrzynki near Żnin, Kujawsko-Pomorskie: B+ (14.09.2013); CC45 Buszkowo near Łabiszyn, Kujawsko-Pomorskie: B+ (15.09.2013); CC45 Łabiszyn, Kujawsko-Pomorskie: B+, Cr (05.05.2006); CC45 Nowe Dąbie near Łabiszyn, Kujawsko-Pomorskie: B+ (05.05.2006); CC46 Lisewo Kościelne near Złotniki Kujawskie, Kujawsko-Pomorskie: B+ (21.08.2008); CC46 Pęchowo near Złotniki Kujawskie, Kujawsko-Pomorskie: B+ (21.08.2008); CC48 Glinno Wielkie near Rojewo, Kujawsko-Pomorskie: B+ (17.08.2008); CC48 Jaszczółtowo near Rojewo, Kujawsko-Pomorskie: B+ (15.08.2008); CC48 Osieczek near Rojewo, KujawskoPomorskie: B+, Cr (17.08.2008); CC48 Osiek near Rojewo, Kujawsko-Pomorskie: B+ (17.08.2008); CC50 Bartodzieje near Wągrowiec, Wielkopolskie: B+ (24.04.2014); CC51 Kopaszyn near Wągrowiec, Wielkopolskie: B+ (01.09.2005); CC51 Werkowo near Wągrowiec, Wielkopolskie: B+ (26.04.2014); CC52 Niemczyn near Damasławek, Wielkopolskie: B+ (26.04.2014); CC52 Wiśniewko near Damasławek, Wielkopolskie: B+ (25.04.2014); CC53 Chrzanowo near Janowiec Wlkp., Kujawsko-Pomorskie: B1.1 (22.05.2014); CC54 Bożejewice near Żnin, Kujawsko-Pomorskie: B1.1 (22.05.2014); CC54 Murczynek near Żnin, Kujawsko-Pomorskie: B+ (15.09.2013); CC55 Barcin, Kujawsko-Pomorskie: B+ (09.05.2008); CC55 Pturek near Barcin, Kujawsko-Pomorskie: B+ (23.05.2014); CC56 Dźwierzchno near Złotniki Kujawskie, Kujawsko-Pomorskie: B+ (13.09.2013); CC56 Sadłogoszcz near Barcin, Kujawsko-Pomorskie: B+ (09.05.2008); CC59 Kijewo near Gniewkowo, Kujawsko-Pomorskie: B+ (18.07.2008); CC59 Murzyńsko near Gniewkowo, Kujawsko-Pomorskie: B+ (19.07.2008); CC59 Szpital near Gniewkowo, Kujawsko-Pomorskie: B+ (18.07.2008); CC59 Wonorze near Dąbrowa Biskupia, Kujawsko-Pomorskie: B+ (18.07.2008); CC60 Runowo near Wągrowiec, Wielkopolskie: B1.1 (04.09.2005); CC60 Runowskie near Wągrowiec, Wielkopolskie: B+ (07.05.2014); CC61 Sarbia near Mieścisko, Wielkopolskie: B+ (27.04.2014); CC61 Wiela near Mieścisko, Wielkopolskie: B+ (23.08.2005); CC61 Zbietka near Mieścisko, Wielkopolskie: B+ (23.08.2005); CC62 Górka near Mieścisko, Wielkopolskie: B1.1 (21.05.2014); CC62 Gruntowice near Damasławek, Wielkopolskie: B1.1 (26.04.2014); CC63 Rogowo, Kujawsko-Pomorskie: B+ (19.05.2014); CC63 Skórki near Rogowo, Kujawsko-Pomorskie: B+ (22.05.2014); CC63 Szkółki near Rogowo, Kujawsko-Pomorskie: B+ (25.08.2005); CC63 Wiewiórczyn near Rogowo, Kujawsko-Pomorskie: B+ (22.05.2014); CC63 Zrazim near Janowiec Wlkp., Kujawsko-Pomorskie: B1.1 (22.05.2014); CC65 Laski Małe near Gąsawa, Kujawsko-Pomorskie: B+ (23.05.2014); CC65 Mokre near Dąbrowa, Kujawsko-Pomorskie: B+ (12.09.2013);row CC65 Obudno near Gąsawa, Kujawsko-Pomorskie: B+ (11.09.2013); CC65 Sławoszewko near Dąbrowa, Kujawsko-Pomorskie: B+ (12.09.2013); CC66 Mierucin near Dąbrowa, Kujawsko-Pomorskie: B+ (12.09.2013); CC70 Grzybowo near Skoki, Wielkopolskie: B+ (24.04.2014); CC70 Skoki, Wielkopolskie: B+ (24.08.2005); CC70 Smolarki near Skoki, Wielkopolskie: B+ (24.08.2005); CC71 Chociszewo near Skoki, Wielkopolskie: B+ (27.04.2014); CC71 Kamieniec near Kłecko, Wielkopolskie: B+ (20.05.2014); CC71 Popowo Kościelne near Mieścisko, Wielkopolskie: B+ (27.04.2014); CC72 Jaroszewo I near Mieścisko, Wielkopolskie: B+ (14.05.2014); CC72 Karniszewo near Mieleszyn, Wielkopolskie: B+ (19.08.2005); CC72 Kłodzin near Mieścisko, 
Wielkopolskie: B+ (22.08.2005); CC72 Łopienno near Mieleszyn, Wielkopolskie: B+ (26.04.2014); CC72 Mieleszyn, Wielkopolskie: B+ (19.05.2014); CC72 Polska Wieś near Kłecko, Wielkopolskie: B1.1 (20.03.2015); CC72 Świniary Dolne near Kłecko, Wielkopolskie: B+ (21.08.2005); CC72 Ułanowo-Folwark near Kłecko, Wielkopolskie: B+ (26.04.2014); CC73 Gącz near Janowiec Wlkp., Kujawsko-Pomorskie: B1.1 (19.05.2014); CC73 Popowo Tomkowe near Mieleszyn, Wielkopolskie: B+, Cr (19.05.2014); CC73 Recz near Rogowo, Kujawsko-Pomorskie: B+ (21.05.2014); CC74 Huta Palędzka near Mogilno, Kujawsko-Pomorskie: B+ (09.05.2008); CC75 Chabsko near Mogilno, Kujawsko-Pomorskie: B+ (07.09.2013); CC75 Chałupska near Mogilno, Kujawsko-Pomorskie: B+ (10.09.2013); CC75 Niestronno near Mogilno, Kujawsko-Pomorskie: B+ (10.09.2013); CC75 Padniewo near Mogilno, Kujawsko-Pomorskie: B+ (10.05.2008); CC76 Dębina near Janikowo, Kujawsko-Pomorskie: B+ (10.10.2007); CC76 Dębowo near Janikowo, Kujawsko-Pomorskie: B+ (10.10.2007); CC76 Olsza near Mogilno, Kujawsko-Pomorskie: B+ (08.05.2008); CC76 Strzelce Budowanie near Mogilno, Kujawsko-Pomorskie: B+ (10.10.2007); CC76 Szczeglin near Mogilno, Kujawsko-Pomorskie: B+ (09.05.2008); CC76 Wszedzień near Mogilno, Kujawsko-Pomorskie: B+ (10.09.2013); CC78 Grodztwo near Kruszwica, Kujawsko-Pomorskie: B+ (14.07.2008); CC80 Głęboczek near Murowana Goślina, Wielkopolskie: B+ (04.05.2014); CC80 Karczewko near Kiszkowo, Wielkopolskie: B+ (04.05.2014); CC80 Rejowiec near Skoki, Wielkopolskie: B+ (17.08.2005); CC80 Turostówko near Kiszkowo, Wielkopolskie: B+ (04.05.2014); CC81 Latalice near Pobiedziska, Wielkopolskie: B+, Cr (26.03.2015); CC81 Rybno Wlk. near Kiszkowo, Wielkopolskie: B1.1 (20.05.2014); CC81 Sroczyn near Kiszkowo, Wielkopolskie: B+ (04.05.2014); CC81 Turostowo near Kiszkowo, Wielkopolskie: B+ (16.08.2005); CC81 Węgorzewo near Kiszkowo, Wielkopolskie: B+ (04.05.2014); CC82 Strychowo near Łubowo, Wielkopolskie: B+ (19.08.2005); CC82 Waliszewo near Kłecko, Wielkopolskie: B+ (18.08.2005); CC83 Gniezno, Wielkopolskie: B+ (04.09.2013); CC83 Goślinowo near Gniezno, Wielkopolskie: B+ (28.06.2008); CC84 Dąbrowa near Trzemeszno, Wielkopolskie: B+ (11.05.2008); CC84 Jastrzębowo near Trzemeszno, Wielkopolskie: B+ (08.09.2013); CC85 Ganina near Gniezno, Wielkopolskie: B+ (11.05.2008); CC85 Wasilewko near Mogilno, Kujawsko-Pomorskie: B+ (12.05.2008); CC86 Kwieciszewo near Mogilno, Kujawsko-Pomorskie: B+ (11.10.2007); CC90 Gorzkie Pole near Pobiedziska, Wielkopolskie: B+ (14.08.2008); CC90 Jerzykowo near Pobiedziska, Wielkopolskie: B+ (14.08.2008); CC90 Kołata near Pobiedziska, Wielkopolskie: B+ (13.08.2005); CC90 Promienko near Pobiedziska, Wielkopolskie: B+ (12.08.2005); CC90 Uzarzewo Huby near Swarzędz, Wielkopolskie: B+ (14.08.2008); CC90 Uzarzewo near Swarzędz, Wielkopolskie: B+ (14.08.2008); CC91 Gołunin near Pobiedziska, Wielkopolskie: B+ (11.08.2005); CC91 Jerzyn near Pobiedziska, Wielkopolskie: B+ (13.08.2005); CC91 Lednogóra near Łubowo, Wielkopolskie: B+ (19.08.2005); CC91 Pobiedziska, Wielkopolskie: B+ (26.03.2013); CC91 Wójtostwo near Pobiedziska, Wielkopolskie: B+ (11.08.2005); CC92 Imielno near Łubowo, Wielkopolskie: B+ (08.08.2005); CC92 Przyborowo near Łubowo, Wielkopolskie: B+ (08.08.2005); CC92 Wierzyce near Łubowo, Wielkopolskie: B+ (08.08.2005); CC93 Drachowo near Niechanowo, Wielkopolskie: B+ (10.08.2005); CC93 Gębarzewo near Czerniejewo, Wielkopolskie: B+ (10.08.2005); CC93 Mnichowo near Gniezno, Wielkopolskie: B+, Cr (10.08.2005); CC93 Żydowo near Czerniejewo, Wielkopolskie: B+ (04.09.2013); CC94 Witkowo, Wielkopolskie: B+ (05.09.2013); CC96 Anastazewo near Powidz, Wielkopolskie: B+ (08.05.2008); CC96 Myślątkowo near Orchowo, Wielkopolskie: B+ (08.05.2008); CC96 Orchowo, Wielkopolskie: B+ (08.05.2008); CC96 Orchówek near Orchowo, Wielkopolskie: B+ (13.06.2008); CC96 Siedluchna near Orchowo, Wielkopolskie: B1 (06.09.2013); CC97 Maślaki near Wilczyn, Wielkopolskie: B+ (13.10.2007); CC98 Wandowo near Skulsk, Wielkopolskie: B+, Cr (08.10.2007); CD00 Łowęcin near Swarzędz, Wielkopolskie: B+ (14.08.2008); CD00 Sokolniki Gwiazdowskie near Swarzędz, Wielkopolskie: B+ (31.03.2015); CD00 Swarzędz, Wielkopolskie: B+ (23.07.2010); CD01 Glinka Duchowna near Kostrzyn Wlkp., Wielkopolskie: B+ (07.08.2005); CD01 Glinka Szlachecka near Kostrzyn Wlkp., Wielkopolskie: B+ (14.08.2008); CD01 Nekielka 
near Nekla, Wielkopolskie: B+ (03.08.2005); CD02 Barczyzna near Nekla, Wielkopolskie: B+ (04.08.2005); CD02 Nekla, Wielkopolskie: B+ (05.08.2005); CD03 Grzybowo near Września, Wielkopolskie: B+ (05.05.2013); CD03 Sobiesiernie near Września, Wielkopolskie: B+ (04.09.2013); CD05 Kochowo near Słupca, Wielkopolskie: B+ (13.06.2008); CD05 Polanowo near Powidz, Wielkopolskie: B+ (13.06.2008); CD07 Kaliska near Wilczyn, Wielkopolskie: B+ (13.10.2007); CD09 Sompolno, Wielkopolskie: B+ (16.06.2008); CD10 Kleszczewo, Wielkopolskie: B+ (11.06.2005); CD10 Strumiany near Borek Wlkp., Wielkopolskie: B+ (13.06.2005); CD10 Trzek near Kostrzyn Wlkp., Wielkopolskie: B+ (31.03.2015); CD18 Izabelin near Kramsk, Wielkopolskie: B+ (21.05.2008); CD19 Pąchów near Kramsk, Wielkopolskie: B+ (22.05.2008); CD22 Miłosław, Wielkopolskie: B+ (12.05.2005); CD24 Pyzdry, Wielkopolskie: B+ (12.05.2005); CD25 Olchowo near Zagórów, Wielkopolskie: B+ (13.05.2005); CD27 Stary Konin near Konin, Wielkopolskie: B+ (23.06.2012); CD27 Węglewskie Holendry near Golina, Wielkopolskie: B+ (04.07.2008); CD29 Tury near Kościelec, Wielkopolskie: B+ (08.06.2008); CD31 Sulęcinek, near Krzykosy, Wielkopolskie: B1.1 (11.05.2005); CD35 Michalinów Oleśnicki near Zagórów, Wielkopolskie: B+ (16.05.2005); CD38 Tuliszków, Wielkopolskie: B+ (25.06.2015); CD40 Książ Wlkp., Wielkopolskie: B+ (15.06.2005); CD45 Orlina Duża near Gizałki, Wielkopolskie: B+ (14.05.2005); CD46 Stare Grądy near Grodziec, Wielkopolskie: B+ (29.09.2007); CD49 Turek, Wielkopolskie: B+ (28.09.2007); CD50 Borek Wlkp., Wielkopolskie: B+ (13.06.2005); CD51 Jaraczewo, Wielkopolskie: B1.1 (13.06.2005); CD51 Łowęcice near Jaraczewo, Wielkopolskie: B+ (13.06.2005); CD53 Wyszogotówek near Kotlin, Wielkopolskie: B+ (24.06.2008); CD56 Stawiszyn, Wielkopolskie: B1.1 (26.05.2005); CD57 Korzeniów near Mycielin, Wielkopolskie: B+ (26.05.2005); CD57 Zbiersk near Stawiszyn, Wielkopolskie: B+ (26.05.2005); CD58 Celestyny near Malanów, Wielkopolskie: B+ (24.09.2007); CD58 Poroże near Malanów, Wielkopolskie: B+ (25.09.2007); CD60 Pogorzela, Wielkopolskie: B+ (01.06.2005); CD60 Siedmiorogów near Borek Wlkp., Wielkopolskie: B+ (01.06.2005); CD63 Izbiczno near Dobrzyca, Wielkopolskie: B+ (21.04.2005); CD63 Karminiec near Dobrzyca, Wielkopolskie: B+ (21.04.2005); CD63 Trzebin near Dobrzyca, Wielkopolskie: B+ (21.04.2005); CD64 Kowalew near Pleszew, Wielkopolskie: B2.2 (21.04.2005); CD67 Aleksandrów near Ceków, Wielkopolskie: B+ (28.05.2005); CD68 Kuźnica near Ceków, Wielkopolskie: B+ (25.09.2007); CD70 Gumienice near Pogorzela, Wielkopolskie: B+ (01.06.2005); CD72 Henryków near Rozdrażew, Wielkopolskie: B+ (05.06.2005); CD73 Koźminiec near Dobrzyca, Wielkopolskie: B1.1 (12.04.2005); CD74 Bieganin near Raszków, Wielkopolskie: B+ (07.05.2005); CD78 Koźminek, Wielkopolskie: B+(22.09.2007); CD81 Dziewiąte near Zduny, Wielkopolskie: B+(31.05.2005); CD81 Kolęda near Zduny, Wielkopolskie: B+ (17.06.2005); CD82 Krotoszyn, Wielkopolskie: B+ (30.05.2005); CD83 Grabów near Ostrów Wlkp., Wielkopolskie: B+ (07.05.2005); CD84 Ostrów Wlkp., Wielkopolskie: B+ (14.06.2005); CD85 Latowice near Sieroszewice, Wielkopolskie: B+ (18.09.2007); CD89 Błaszki, Łódzkie: B+ (19.09.2007); CD91 Cieszków, Dolnośląskie: B+ (17.06.2005); CD91 Grzebielin near Milicz, Dolnośląskie: B+ (20.06.2005); CD92 Bartniki near Milicz, Dolnośląskie: B+ (19.06.2005); CD92 Joachimówka near Milicz, Dolnośląskie: B+ (31.07.2010); CD92 Kolęda near Milicz, Dolnośląskie: B+ (14.06.2005); CD92 Latkowa near Milicz, Dolnośląskie: B+ (19.06.2005); CD95 Kotłów near Mikstat, Wielkopolskie: B+ (06.08.2010); CD96 Kalisz, Wielkopolskie: B+ (16.05.2008); CD96 Ołobok near Sieroszewice, Wielkopolskie: B+ (19.09.2007); CD98 Joanka near Szczytniki, Wielkopolskie: B+ (19.09.2007); CE00 Postolin near Milicz, Dolnośląskie: B+ (27.07.2010); CE00 Pracze near Milicz, Dolnośląskie: B+ (27.07.2010); CE01 Dąbrowa near Krośnice, Dolnośląskie: B+ (29.07.2010); CE01 Duchowa near Milicz, Dolnośląskie: B+ (29.07.2010); CE01 Grabownica near Milicz, Dolnośląskie: B+ (26.07.2010); CE01 Milicz, Dolnośląskie: B+ (01.05.2014); CE01 Wąbnice near Krośnice, Dolnośląskie: B+ (29.07.2010); CE02 Luboradów near Krośnice, Dolnośląskie: B+ (31.07.2010); CE03 Granowiec near Sośnie, Wielkopolskie: B+ (01.08.2010); CE03 Janisławice near Sośnie, Wielkopolskie: B+ (01.08.2010); CE04 Szklarka Przygodzicka near Ostrzeszów, Wielkopolskie: 
B+ (07.08.2010); CE05 Niedźwiedź near Ostrzeszów, Wielkopolskie: B+ (04.05.2011); CE07 Grabów on the Prosna, Wielkopolskie: B+ (20.09.2007); CE11 Brzostowo near Krośnice, Dolnośląskie: B+ (30.07.2010); CE11 Bukowice near Krośnice, Dolnośląskie: B+ (22.07.2010); CE11 Lędzina near Krośnice, Dolnośląskie: B+ (26.04.2011); CE11 Maleszów near Dobroszyce, Dolnośląskie: B+ (24.04.2011); CE11 Miłochowice near Milicz, Dolnośląskie: B+ (25.07.2010); CE11 Pierstnica near Krośnice, Dolnośląskie: B+ (30.07.2010); CE12 Moszyce near Twardogóra, Dolnośląskie: B+ (29.04.2011); CE13 Chojnik near Sośnie, Wielkopolskie: B+ (07.08.2010); CE13 Kraszów near Międzybórz, Dolnośląskie: B+ (09.08.2010); CE13 Ligota Rybińska near Międzybórz, Dolnośląskie: B+ (08.08.2010); CE13 Międzybórz, Dolnośląskie: B+ (08.08.2010); CE14 Kobyla Góra, Wielkopolskie: B+ (09.08.2010); CE14 Kuźnica Myślniewska near Kobyla Góra, Wielkopolskie: B+ (08.08.2010); CE14 Marcinki near Kobyla Góra, Wielkopolskie: B+ (09.08.2010); CE23 Dziesławice near Międzybórz, Dolnośląskie: B+ (02.08.2010); CE26 Wieruszów, Łódzkie: B+ (10.08.2010); CE92 Potasznia near Milicz, Dolnośląskie: B+ (23.04.2011); CE92 Sulmierzyce, Wielkopolskie: B+ (28.07.2010); CE93 Uciechów near Odolanów, Wielkopolskie: B+ (04.08.2010); DC40 Brzoza near Wielka Nieszawka, Kujawsko-Pomorskie: B+ (17.07.2008); DC51 Ciechocinek, Kujawsko-Pomorskie: B+ (15.07.2008); DC51 Nowy Ciechocinek near Aleksandrów Kujawski, Kujawsko-Pomorskie: B+ (15.07.2008); DC51 Raciążek, Kujawsko-Pomorskie: B+ (15.07.2008); DC61 Kamieniec near Koneck, Kujawsko-Pomorskie: B+ (16.07.2008); DC62 Stary Zbrachlin near Waganiec, Kujawsko-Pomorskie: B+ (20.06.2008); DC80 Czarnocice near Bytoń, Kujawsko-Pomorskie: B+ (14.07.2008); DC81 Łąkowa Wola near Brześć Kujawski, Kujawsko-Pomorskie: B+ (17.06.2008); DD00 Zaryń near Wierzbinek, Wielkopolskie: B+ (16.06.2008); DD01 Izbica Kujawska, Kujawsko-Pomorskie: B+ (16.06.2008); DD01 Tymień near Izbica Kujawska, Kujawsko-Pomorskie: B+ (15.06.2008); DD10 Borecznia Wlk. near Osiek Mały, Wielkopolskie: B+ (23.05.2008); DD10 Lipiny near Sompolno, Wielkopolskie: B+ (23.05.2008); DD11 Rysiny Kol. near Kłodawa, Wielkopolskie: B+ (27.07.2012); DD20 Koło, Wielkopolskie: B+ (26.06.2015); DD20 Osiek Wielki near Osiek Mały, Wielkopolskie: B+ (28.06.2008); DD21 Zawady Nowe near Olszówki, Wielkopolskie: B+ (28.05.2008); DD22 Kłodawa, Wielkopolskie: B+ (26.07.2012); DD22 Smardzew near Grabów, Łódzkie: B+ (28.05.2008); DD22 Zawadka near Olszówka, Wielkopolskie: B+ (25.07.2012); DD30 Daniszew near Kościelec, Wielkopolskie: B+ (27.09.2007); DD30 Łąka near Przytoka, Wielkopolskie: B+ (27.09.2007); DD31 Dąbie, Wielkopolskie: B1.1 (23.06.2012); DD31 Łusie near Dąbie, Wielkopolskie: B+ (26.05.2005); DD31 Szczepanów near Olszówka, Wielkopolskie: B+ (26.05.2008); DD32 Baranowiec near Dąbie, Wielkopolskie: B+ (28.05.2008); DD40 Sarbice near Kościelec, Wielkopolskie: B+ (01.05.2014); DD41 Dąbrowa near Uniejów, Łódzkie: B+ (27.05.2008); DD41 Wielenin near Uniejów, Łódzkie: B+ (27.05.2008); DD70 Warta, Łódzkie: B+ (22.09.2007).

Rosa canina L. $\times$ R. glauca Pourret (leg. A. Czarna, det. J. Zieliński)

CD09 Sompolno, Wielkopolskie: B+ (16.06.2008).

Rosa canina L. $\times$ R. rubiginosa L. (leg. A. Czarna, det. J. Zieliński)

BC69 Potuły near Wągrowiec, Wielkopolskie: B+ (07.05.2014).

Rosa dumalis Bechst. (leg. det. A. Czarna, with few exceptions)

AC85 Dzierzążna near Krzeszyce, Lubuskie: B+ (10.09.2006); AD35 Korczyców near Maszewo, Lubuskie: B+ (03.06.2010); AD55 Wełmica near Bobrowice, Lubuskie: B1.1 (26.03.2014); AD67 Koźla near Świdnica, Lubuskie: B+ (27.08.2008); AD78 Chotków near Brzeźnica, Lubuskie: B+ (14.09.2008); AD79 Stypułów near Kożuchów, Lubuskie: B+ (14.09.2008); BC46 Zofiewo near Czarnków, Wielkopolskie: B+ (09.05.2014); BD70 Kiełcz near Nowa Sól, Lubuskie: B+ (09.09.2008); BD81 Gostyń near Gaworzyce, Dolnośląskie: B+ (29.08.2009); BD81 
Zimna Brzeźnica near Niegosławice, Lubuskie: B+ (22.08.2009); CC46 Pęchowo near Złotniki Kujawskie, Kujawsko-Pomorskie: B+ (21.08.2008); CC50 Bartodzieje near Wągrowiec, Wielkopolskie: B+ (24.04.2014); CC54 Bożejewice near Żnin, Kujawsko-Pomorskie: B+ (14.05.2006). Miąskowo near Kcynia, Kujawsko-Pomorskie: B+ (05.2014); CC55 Barcin, Kujawsko-Pomorskie: B+ (09.05.2008); CC63 Zrazim near Janowiec Wlkp., Kujawsko-Pomorskie: B+ (leg. A. Czarna, det. J. Zieliński,22.05.2014); CC82 Strychowo near Łubowo, Wielkopolskie: B+ (leg. A. Czarna, det. J. Zieliński, 19.08.2005); CC93 Drachowo near Niechanowo, Wielkopolskie: B+ (leg. A. Czarna, det. J. Zieliński,10.08.2005); CD02 Barczyzna near Nekla, Wielkopolskie: B+ (04.08.2005); DD21 Nowa Zawadka near Olszówka, Wielkopolskie: B+ (25.07.2012).

Rosa inodora Fries (leg. A. Czarna, det. J. Zieliński)

CC81 Rybno Wielkie near Kiszkowo, Wielkopolskie: B+ (21.08.2005).

Rosa inodora Fries $\times \boldsymbol{R}$. rubiginosa L. (leg. A. Czarna, det. J. Zieliński)

BD82 Kurowice near Jerzmanowa, Dolnośląskie: B1.1 (28.08.2009).

Rosa jundzillii Besser (leg. det. A. Czarna)

CC46 Pęchowo near Złotniki Kujawskie, Kujawsko-Pomorskie: B+ (21.08.2008).

Rosa rubiginosa L. (leg. det. A. Czarna, with few exceptions)

AC59 Górki Noteckie near Zwierzyn, Lubuskie: B1.1 (leg. A. Czarna, det. J. Zieliński, 08.03.2015); AC84 Słońsk, Lubuskie: B+ (29.05.2010); AC97 Lubniewice, Lubuskie: B+ (21.09.2006); AD48 Wysokie near Czerwieńsk, Lubuskie: B+ (09.06.2010); BC42 Brzegi near Krzyż Wlkp., Wielkopolskie: B+ (05.09.2011); BC59 Brzekiniec near Wągrowiec, Wielkopolskie: B+ (02.09.2005); BC69 Potuly near Wągrowiec, Wielkopolskie: B+ (07.05.2014); BC82 Kolno near Międzychód, Wielkopolskie: B+ (10.04.2010); BD76 Czernina near Góra, Dolnośląskie: B+ (04.06.2005); BD95 Smolno near Jemielno, Dolnośląskie: B1.1 (03.09.2009); BD98 Szymanowo near Rawicz, Wielkopolskie: B+ (29.06.2005); CC45 Buszkowo near Łabiszyn, Kujawsko-Pomorskie: B+ (15.09.2013); CC63 Zrazim near Janowiec Wlkp., Kujawsko-Pomorskie: B+ (22.05.2014); CC70 Budziszewko near Rogoźno Wlkp., Wielkopolskie: B+ (28.08.2005); CC72 Łopienno near Mieleszyn, Wielkopolskie: B+ (26.04.2014); CC81 Rybno Wlk. near Kiszkowo, Wielkopolskie: B1.1 (20.05.2014); CE01 Pracze near Milicz, Dolnośląskie: B+ (leg. A. Czarna, det. J. Zieliński, 7.07.2010); DC51 Raciążek, Kujawsko-Pomorskie: B + (leg. A. Czarna, det. J. Zieliński, 15.07.2008).

Rosa rubiginosa $\times$ R. sherardii Davies (leg. A. Czarna, det. J. Zieliński)

BC26 Herbutowo near Wieleń, Wielkopolskie: B1.1 (09.03.2015).

Rosa sherardii Davies (leg. det. A. Czarna, with few exceptions)

AC53 Kostrzyn on the Oder, Lubuskie: B+ (04.03.2015); AC77 Glinik near Deszczno, Lubuskie: B+ (11.05.2010); AC84 Słońsk, Lubuskie: B+ (leg. A. Czarna, det. J. Zieliński, 13.09.2006); AC89 Skrzynica near Skwierzyna, Lubuskie: B+ (28.04.2010); AC96 Miechów near Sulęcin, Lubuskie: B+ (leg. A. Czarna, det. J. Zieliński,10.09.2006); AC97 Trzemeszno Lubuskie near Sulęcin, Lubuskie: B+ (21.09.2006); AD04 Rzepin, Lubuskie: B+ (14.09.2006); AD44 Drzeńsk Wielki near Gubin, Lubuskie: B+ (14.06.2010); AD45 Chojna near Krosno Odrzańskie, Lubuskie: B+ (13.06.2010); AD54 Kaniów near Gubin, Lubuskie: B+ (19.09.2008); AD55 Przychów near Bobrowice, Lubuskie: B+ (21.09.2008); AD58 Świdnica, near Świdnica, Zielona Góra, Lubuskie: B+ (27.08.2008); AD63 Jasienica near Brody, Lubuskie: B+ (20.09.2008); AD64 Raszyn near Lubsko, Lubuskie: B1.1 (27.03.2014); AD65 Tymienice near Lubsko, Lubuskie: B+ (leg. A. Czarna, 
det. J. Zieliński, 26.03.2014); AD74 Tuplice, Lubuskie: B+ (31.03.2014); AD75 Golin near Jasień, Lubuskie: B1.1 (16.09.2008); AD79 Leśniów Wielki near Czerwieńsk, Lubuskie: B+ (leg. A. Czarna, det. J. Zieliński, 27.08.2008); AD84 Tuplice, Lubuskie: B+ (31.03.2014); BC26 Herbutowo near Wieleń, Wielkopolskie: B1.1 (09.03.2015); BC26 Łomnica-Młyn near Trzcianka, Wielkopolskie: B+ (12.03.2015); BC42 Brzegi near Krzyż Wlkp., Wielkopolskie: B+ (05.09.2011); BC43 Lubcz Mały near Krzyż Wlkp., Wielkopolskie: B+ (05.09.2011); BC50 Przynotecko near Stare Kurowo, Lubuskie: B+ (07.05.2013); BC51 Drezdenko, Lubuskie: B+ (15.04.2010); BC58 Wyszynki near Budzyń, Wielkopolskie: B+ (25.04.2006); BC60 Gościm near Drezdenko, Lubuskie: B+ (11.08.2006); BC69 Rogoźno Wlkp., Wielkopolskie: B+ (23.04.2014); BC76 Jaryszewo near Obrzycko, Wielkopolskie: B+ (leg. A. Czarna, det. J. Zieliński,20.07.2006); BC76 Piotrkówki near Szamotuły, Wielkopolskie: B+ (08.05.2007); BC78 Łukowo near Oborniki, Wielkopolskie: B+ (15.09.2007); BC78 Słonawy near Oborniki, Wielkopolskie: B+ (15.09.2007); BD31 Kopanica near Siedlec, Wielkopolskie: B+ (18.04.2007); BD53 Śmieszkowo near Sława, Lubuskie: B+ (18.10.2011); BD67 Rydzyna, Wielkopolskie: B+ (04.06.2005); BD76 Czernina near Góra, Dolnośląskie: B1.1 (04.06.2005); CC44 Ciążkowo near Szubin, Kujawsko-Pomorskie: B+ (03.05.2006); CC44 Wawrzynki near Żnin, Kujawsko-Pomorskie: B+ (04.05.2006); CC45 Buszkowo near Łabiszyn, Kujawsko-Pomorskie: B+ (04.05.2006); CC48 Jaszczołtowo near Rojewo, Wielkopolskie: B+ (15.08.2008); CC50 Bartodzieje near Wągrowiec, Wielkopolskie: B1.1 (24.04.2014); CC54 Wilczkowo near Żnin, Kujawsko-Pomorskie: B+ (04.05.2006); CC55 Barcin, Kujawsko-Pomorskie: B+ (09.05.2008); CC59 Wonorze near Dąbrowa Biskupia, Kujawsko-Pomorskie: B+ (18.07.2008); CC61 Sarbia near Mieścisko, Wielkopolskie: B+ (27.04.2014); CC71 Łęczno near Miedzichowo, Wielkopolskie: B+ (05.09.2006); CC72 Jaroszewo I near Mieścisko, Wielkopolskie: B+ (14.05.2014); CC73 Gącz near Janowiec Wlkp., Kujawsko-Pomorskie: B+ (25.08.2005); CC73 Popowo Tomkowe near Mieleszyn, Wielkopolskie: B+ (22.05.2014); CC76 Strzelce Budowanie near Mogilno, Kujawsko-Pomorskie: B+ (10.10.2007); CC94 Witkowo, Wielkopolskie: B+ (05.09.2013); CD07 Kaliska near Wilczyn, Wielkopolskie: B+ (13.10.2007); CD18 Izabelin near Kramsk, Wielkopolskie: B+ (21.05.2008); CD78 Koźminek, Wielkopolskie: B+ (22.09.2007); CE03 Janisławice near Sośnie, Wielkopolskie: B+ (01.08.2010); DD02 Cetty near Chodecz, Kujawsko-Pomorskie: B+ (18.06.2008); DD10 Lipiny near Sompolno, Wielkopolskie: B+ (leg. A. Czarna, det. J. Zieliński, 23.05.2008).

Rosa tomentosa Smith (leg. A. Czarna, det. J. Zieliński)

AD25 Bargów near Torzym, Lubuskie: B+ (26.09.2006).

Rosa villosa L. (leg. det. A. Czarna)

AC77 Rudnica near Krzeszyce, Lubuskie: B+ (20.09.2006); AC94 Ośno Lubuskie, Lubuskie: B+ (12.09.2006); BC50 Trzebicz near Drezdenko, Lubuskie: B+ (15.04.2010); BC52 Chełst near Krzyż Wlkp. Drawsko, Wielkopolskie: B+ (10.08.2006); BC58 Ryczywół, Wielkopolskie: B+ (22.09.2011); CC25 Białe Błota, Kujawsko-Pomorskie: B+ (23.08.2008).

Rosa zalana Wiesb. (leg. A. Czarna, det. J. Zieliński)

CD83 Grabów near Ostrów Wlkp., Wielkopolskie: B+ (07.05.2005).

\section{LIST OF ALIEN TAXA OF THE GENUS ROSA}

Rosa xalba L. (leg. A. Czarna, det. J. Zieliński)

AC53 Kostrzyn on the Oder, Lubuskie: B1.1 (04.03.2015); AD03 Lisów near Słubice, Lubuskie: B+ (31.05.2010); AD25 Gądków Wielki near Torzym, Lubuskie: B+ (02.06.2010); BC17 Piła, Wielkopolskie: B+ (29.04.2014); BC36 Biała near Trzcianka, Wielkopolskie: B1.1 (12.05.2014); BC38 Karolinka near Margonin, Wielkopolskie: B1.1 (16.05.2014); BC38 Nietusz- 
kowo near Chodzież, Wielkopolskie: B1.1 (19.09.2005); BC60 Gościm near Drezdenko, Lubuskie: B+ (11.08.2006); BD76 Sułów near Góra, Dolnośląskie: B+ (04.07.2005); BD95 Kurów Mały near Jerzmanowa, Dolnośląskie: B+ (31.08.2009); CC20 Sokolec near Szamocin, Wielkopolskie: B+ (15.05.2006); CC45 Łabiszyn, Kujawsko-Pomorskie: B1.1 (05.05.2006); CC61 Wiela near Mieścisko, Wielkopolskie: B+ (23.08.2005); CC81 Turostowo near Kiszkowo, Wielkopolskie: B+ (16.08.2005); CD27 Konin, Wielkopolskie: B+ (23.06.2012); CD51 Jaraczewo, Wielkopolskie: B1.1 (13.06.2005); CD76 Kalisz, Wielkopolskie: B+ (27.08.2005); DD22 Stara Zawadka near Olszówka, Wielkopolskie: B+ (25.07.2015).

Rosa blanda Ait. (leg. A. Czarna, det. J. Zieliński)

BD08 Poznań-Górczyn, Wielkopolskie: B1.1 (26.07.2014); CD24 Pyzdry, Wielkopolskie: B1.1 (12.05.2005).

Rosa xcentifolia L. (leg. A. Czarna, det. J. Zieliński)

AD26 Dobrosułów near Bytnica, Lubuskie: B+ (12.06.2010); BC17 Piła, Wielkopolskie: B+ (29.04.2014); BC39 Strzelce near Chodzież, Wielkopolskie: B+ (19.09.2005); BD76 Sułów near Góra, Dolnośląskie: B1.1 (04.07.2005); CC59 Wonorze near Dąbrowa Biskupia, Kujawsko-Pomorskie: B + (18.07.2008); CD10 Strumiany near Borek Wlkp., Wielkopolskie: B1.1 (13.06.2005); DD20 Koło, Wielkopolskie: B+ (26.06.2012).

Rosa xdamascena Mill. (leg. A. Czarna, det. J. Zieliński)

AC69 Jastrzębnik near Santok, Lubuskie: B+ (15.04.2010); AD64 Osiek near Lubsko, Lubuskie: B+ (15.09.2008); AD87 Żagań, Lubuskie: B+ (14.04.2009); BC17 Piła, Wielkopolskie: B+ (29.04.2014); BD82 Żukowice, Dolnośląskie: B+ (10.09.2008); CE02 Luboradów near Krośnice, Dolnośląskie: $\mathrm{B}+(31.07 .2010)$.

Rosa foetida J. Herrmann (leg. A. Czarna, det. J. Zieliński)

BD98 Szymanowo near Rawicz, Wielkopolskie: B+ (26.05.2012); DD22 Smardzew near Grabów, Łódzkie: B1.1 (28.05.2008).

Rosa foliolosa Nutt. (leg. A. Czarna, det. J. Zieliński)

CD76Kalisz, Wielkopolskie: B1.1 (16.05.2008).

Rosa xfrancofourtana Muenchh. (leg. A. Czarna, det. J. Zieliński)

AC67 Osiedle Poznańskie near Deszczno, Lubuskie: B+ (21.09.2006); AC74 Kłopotowo near Witnica, Lubuskie: B1.1 (10.09.2006); AC75 Przemysław near Krzeszyce, Lubuskie: B1.1 (29.05.2010); AC77 Prądocin near Deszczno, Lubuskie: B2.2 (21.09.2006); AC85 Dzierzążna near Krzeszyce, Lubuskie: B2.1 (10.09.2006); AC85 Lemierzyce near Słońsk, Lubuskie: B+ (10.09.2006); AD64 Jałowice near Brody, Lubuskie: B+ (18.09.2008); AD65 Chocicz near Lubsko, Lubuskie: B1.1 (21.09.2008); AD67 Podgórzyce near Nowogród Bobrzański, Lubuskie: B1.1 (31.08.2008); AD78 Broniszów near Kożuchów, Lubuskie: B+ (17.08.2009); BC27 Byszki near Ujście, Wielkopolskie: B+ (23.08.2010); BC38 Karolinka near Margonin, Wielkopolskie: B1.1 (16.05.2014); BC38 Milicz near Chodzież, Wielkopolskie: B+ (01.05.2014); BC38 Studzieniec near Chodzież, Wielkopolskie: B+ (01.05.2014); BC39 Nałęcza near Szamocin, Wielkopolskie: B+ (18.03.2015); BC39 Strzelęcin near Chodzież, Wielkopolskie: B1.1 (02.05.2014); BC47 Paliszewo near Czarnków, Wielkopolskie: B+ (16.07.2006); BC48 Stróżewo near Chodzież, Wielkopolskie: B2.2 (20.05.2006); BC48 Wyszyna near Władysławów, Wielkopolskie: B+ (03.05.2014); BC51 Pełcza near Drezdenko, Lubuskie: B1.1 (10.08.2006); BC79 Budziszewko near Rogoźno Wlkp., Wielkopolskie: B+ (22.04.2014); BC79 Stary Łoskoń near Murowana Goślina, Wielkopolskie: 
B+ (22.04.2014); BC89 Złotoryjsko near Murowana Goślina, Wielkopolskie: B1.1 (16.09.2007); BC99 Owińska near Czerwonak, Wielkopolskie: B+ (04.05.2014); BD07 Zakrzewo near Dopiewo, Wielkopolskie: B+ (01.06.2007); BD31 Kopanica near Siedlec, Wielkopolskie: B+ (18.04.2007); BD40 Drążeń near Krzymów, Wielkopolskie: B1.1 (24.04.2007); BD56 Goniembice near Lipno, Wielkopolskie: B1.1 (20.06.2006); BD70 Nowe Żabno near Nowa Sól, Lubuskie: B+ (09.09.2008); BD72 Kotla, Dolnośląskie: B+ (20.04.2012); BD76 Czernina Górna near Góra, Dolnośląskie: B+ (16.05.2012); BD82 Kurowice near Jerzmanowa, Dolnośląkie: B1.1 (28.08.2009); BD82 Nielubia near Żukowice, Dolnośląskie: B1.1 (29.08.2009); BD85 Strumienne near Góra, Dolnośląskie: B1.1 (05.07.2005); BD94 Kłębów near Rudna, Dolnośląskie: B2.2 (01.09.2009); BD95 Chobieniek near Rudna, Dolnośląskie: B+ (02.09.2009); BD98 Radziądz near Żmigród, Dolnośląskie: B+ (25.07.2010); CC21 Osiek on the Noteć near Wyrzysk, Wielkopolskie: B1.1 (08.09.2011); CC27 Wypaleniska near Solec Kujawski, Kujawsko-Pomorskie: B+ (19.08.2008); CC30 Borowo near Szamocin, Wielkopolskie: B+ (14.05.2006); CC30 Borówki near Szamocin, Wielkopolskie: B+ (15.05.2006); CC30 Szamocin, Wielkopolskie: B+ (14.05.2006); CC31 Nowa Wieś Notecka near Kcynia, Wielkopolskie: B1.1 (14.05.2006); CC33 Słonawy near Szamocin, Wielkopolskie: B+ (10.05.2006); CC35 Władysławowo near Łabiszyn, Kujawsko-Pomorskie: B+ (06.05.2006); CC59 Wonorze near Dąbrowa Biskupia, Kujawsko-Pomorskie: B2.2 (18.07.2008); CC60 Runowo near Wągrowiec, Wielkopolskie: B+ (04.09.2005); CC62 Gruntowice near Damasławek, Wielkopolskie: B1.1 (26.04.2014); CC63 Zrazim near Janowiec Wlkp., Kujawsko-Pomorskie: B+ (22.05.2014); CC70 Smolarki near Skoki, Wielkopolskie: B+ (24.08.2005); CC73 Gącz near Janowiec Wlkp., Kujawsko-Pomorskie: B1.1 (25.08.2005); CC76 Olsza near Mogilno, Kujawsko-Pomorskie: B+ (08.05.2008); CC81 Rybno Wielkie near Kiszkowo, Wielkopolskie: B+ (21.08.2005); CC90 Promienko near Pobiedziska, Wielkopolskie: B1.1 (12.08.2005); CD02 Nekla, Wielkopolskie: B1.1 (05.08.2005); CD09 Zakrzewek near Sompolno, Wielkopolskie: B+ (15.06.2008); CD24 Pyzdry, Wielkopolskie: B+ (12.05.2005); CD27 Konin, Wielkopolskie: B+ (23.06.2012); CD68 Kuźnica near Ceków, Wielkopolskie: B1.1 (25.09.2007); CE02 Luboradów near Krośnice, Dolnośląskie: B+ (31.07.2010); CE12 Moszyce near Twardogóra, Dolnośląskie: B1.1 (29.04.2011); DC61 Kamieniec near Koneck, Kujawsko-Pomorskie: B+ (16.07.2008); DC90 Bycz near Piotrków Kujawski, Kujawsko-Pomorskie: B+ (14.06.2008); DD00 Marcinkowo near Sompolno, Wielkopolskie: B+ (15.06.2008); DD10 Babiak, Wielkopolskie: B+ (15.06.2008); DD10 Kiejsze near Babiak, Wielkopolskie: B+ (27.07.2012); DD10 Siedliska near Sompolno, Wielkopolskie: B+ (15.06.2008); DD21 Nowa Zawadka near Olszówka, Wielkopolskie: B+ (25.07.2012); DD22 Stara Zawadka near Olszówka, Wielkopolskie: B+ (25.07.2012); DD40 Sarbice near Kościelec, Wielkopolskie: B1.1 (01.05.2014); DD41 Dąbrowa near Uniejów, Łódzkie: B+ (27.05.2008).

\section{Rosa gallica L. 'Plena' (leg. A. Czarna, det. J. Zieliński)}

AC54 Ownice near Słońsk, Lubuskie: B1.1 (20.09.2006); AC89 Twierdzielewo near Przytoczna, Lubuskie: B1.1 (08.09.2006); AC95 Radachów near Ośno Lubelskie, Lubuskie: B+ (19.06.2006); AC96 Miechów near Sulęcin, Lubuskie: B1.1 (10.09.2006); AC96 Ostrów near Sulęcin, Lubuskie: B+ (27.05.2010); AC99 Kolsko, Lubuskie: B+ (05.05.2010); AD28 Lubrza, Lubuskie: B+ (23.09.2006); AD35 Skarbona near Maszewo, Lubuskie: B+ (25.09.2006); AD45 Chojna near Krosno Odrzańskie, Lubuskie: B+ (13.06.2010); AD46 Marcinowice near Krosno Odrzańskie, Lubuskie: B+ (21.09.2006); AD53 Bieżyce near Gubin, Lubuskie: B+ (19.09.2008); AD57 Drzonów near Świdnica, Lubuskie: B+ (27.08.2008); AD57 Lipno near Świdnica, Lubuskie: B1.1 (27.08.2008); AD67 Kaczeniec near Nowogród Bobrzański, Lubuskie: B1.1 (29.08.2008); AD69 Czasław near Otyń, Lubuskie: B+ (26.08.2008); AD75 Golin near Jasień, Lubuskie: B1.1 (16.09.2008); AD78 Broniszów near Kożuchów, Lubuskie: B1.1 (17.08.2009); AD85 Górka near Lipinki Łużyckie, Lubuskie: B1.1 (20.05.2009); AD87 Żagań, Lubuskie: B+ (14.04.2009r BC17 Piła, Wielkopolskie: B+ (29.04.2014); BC18 Brodna near Kaczory, Wielkopolskie: B+ (26.08.2010); BC27 Piła, Wielko- 
polskie: B+ (29.04.2014); BC28 Kaczory, Wielkopolskie: B1+ (30.04.2014); BC28 Piła-Leszków, Wielkopolskie: B+ (29.04.2014); BC36 Romanowo Górne near Czarnków, Wielkopolskie: B1.1 (16.07.2006); BC38 Milicz near Chodzież, Wielkopolskie: B1.1 (01.05.2014); BC39 Strzelce near Chodzież, Wielkopolskie: B1.1 (19.09.2005); BC44 Folsztyn near Wieleń, Wielkopolskie: B1.1 (10.05.2014); BC46 Romanowo Dolne near Czarnków, Wielkopolskie: B+ (08.05.2014); BC49 Adolfowo near Margonin, Wielkopolskie: B+ (02.05.2014); BC54 Rosko near Wieleń, Wielkopolskie: B1.1 (07.04.2010); BC59 Brzekiniec near Budzyń, Wielkopolskie: B+ (02.09.2005); BC59 Nowe Brzeźno near Budzyń, Wielkopolskie: B1.1 (02.09.2005); BC60 Gościm near Drezdenko, Lubuskie: B+ (11.08.2006); BC70 Krobielewko near Skwierzyna, Lubuskie: B+ (28.04.2010); BC73 Kaczlin near Sieraków, Wielkopolskie: B1.1 (06.08.2006); BC76 Jaryszewo near Obrzycko, Wielkopolskie: B2.2 (20.07.2006); BC80 Zielomyśl near Pszczew, Lubuskie: B+ (16.08.2006); BC84 Psarki near Pniewy, Wielkopolskie: B1.1 (08.08.2006); BC91 Stoki near Pszczew, Lubuskie: B+ (02.05.2010); ); BD00 Siercz near Trzciel, Lubuskie: B1.1 (07.09.2006); BD04 Jastrzębnik near Opalenica, Wielkopolskie: B+ (20.05.2010); BD06 Buk, Wielkopolskie: B1.1 (31.08.2006); BD41 Bełcze near Bojadła, Lubuskie: B+ (13.09.2008); BD42 Nowy Jaromierz near Kargowa, Lubuskie: B+ (29.04.2007); BD43 Nowa Wieś near Przemęt, Wielkopolskie: B1.1 (07.05.2012); BD76 Sułków near Góra, Dolnośląskie: B+ (16.05.2012); BD84 Bełcz Wielki near Niechlów, Dolnośląskie: B+ (02.09.2009); BD85 Strumienne near Góra, Dolnośląskie: B1.1 (05.07.2005); CC25 Białe Błota, Kujawsko-Pomorskie: B1.1 (23.08.2008); CC41 Laskownica Wielka near Gołańcz, Wielkopolskie: B1.1 (30.08.2005); CC45 Nowe Dąbie near Łabiszyn, Kujawsko-Pomorskie: B1.1 (05.05.2006); CC48 Jaszczołtowo near Rojewo, Kujawsko-Pomorskie: B+ (15.08.2008); CC59 Wonorze near Dąbrowa Biskupia, Kujawsko-Pomorskie: B+ (18.07.2008); CC61 Sarbia near Mieścisko, Wielkopolskie: B+ (29.08.2005); CC72 Jaroszewo near Mieścisko, Wielkopolskie: B+ (14.05.2014); CC72 Polska Wieś near Kłecko, Wielkopolskie: B1.1 (21.08.2005); CC72 Ułanowo-Folwark near Kłecko, Wielkopolskie: B1.1 (26.04.2014); CC76 Dębina near Janikowo, Kujawsko-Pomorskie: B+ (10.10.2007); CC81 Rybno Wielkie near Kiszkowo, Wielkopolskie: B+ (21.08.2005); CC81 Turostowo near Kiszkowo, Wielkopolskie: B1.1 (16.08.2005); CC82 Strychowo near Łubowo, Wielkopolskie: B1.1 (19.08.2005); CC92 Imielno near Łubowo, Wielkopolskie: B1.1 (08.08.2005); CC92 Przyborowo near Łubowo, Wielkopolskie: B1.1 (08.08.2005); CD00 Łowęcin near Swarzędz, Wielkopolskie: B1.1 (14.08.2008); CD02 Barczyzna near Nekla, Wielkopolskie: B1.1 (04.08.2005); CD51 Łowęcice near Jaraczewo, Wielkopolskie: B+ (13.06.2005); CD78 Koźminek, Wielkopolskie: B1.1 (22.09.2007); CD85 Latowice near Sieroszewice, Wielkopolskie: B1.1 (18.09.2007); CD95 Kotłów near Mikstat, Wielkopolskie: B+ (06.08.2010); CD96 Ołobok near Sieroszewice, Wielkopolskie: B+ (19.09.2007); CE02 Luboradów near Krośnice, Dolnośląskie: B+ (31.07.2010); CE07 Grabów on the Prosna, Wielkopolskie: B+ (20.09.2007); CE92 Sulmierzyce, Wielkopolskie: B1.1 (28.07.2010); DC51 Nowy Ciechocinek near Aleksandrów Kujawski, Kujawsko-Pomorskie: B2.2 (15.07.2008); DC61 Kamieniec near Koneck, Kujawsko-Pomorskie: B+ (16.07.2008); DD02 Przysypka near Chodecz, Kujawsko-Pomorskie: B1.1 (18.06.2008); DD22 Zawadka near Olszówka, Wielkopolskie: B1.1 (25.07.2012).

Rosa gallica $\times$ R. dumalis (leg. A. Czarna, det. J. Zieliński)

BC17 Piła, Wielkopolskie: B+ (29.04.2014).

Rosa glauca Pourret (leg. A. Czarna, det. J. Zieliński)

CD09 Sompolno, Wielkopolskie: B+ (16.06.2008).

Rosa gorenkensis Besser (leg. A. Czarna, det. J. Zieliński)

AC96 Sulęcin, Lubuskie: B+ (11.09.2006); AD58 Świdnica, Lubuskie: B+ (27.08.2008); CE11 Brzostowo near Krośnice, Dolnośląskie: B+ (30.07.2010); CE14 Kobyla Góra, Wielkopolskie: $\mathrm{B}+(09.08 .2010)$. 
Rosa majalis J. Herrmann 'Foecundissima' (leg. A. Czarna, det. J. Zieliński, with exceptions)

AC69 Jastrzębnik near Santok, Wielkopolskie: B1.1 (15.04.2010); AD15 Lubin near Torzym, Lubuskie: B+ (leg. det. A. Czarna, 02.06.2010); AD26 Dobrosułów near Bytnica, Lubuskie: B+ (leg. det. A. Czarna, 12.06.2010); AD36 Bytnica, Lubuskie: B1.1 (24.09.2006); AD55 Wełmica near Bobrowice, Lubuskie: B1.1 (leg. det. A. Czarna, 26.03.2014); AD58 Świdnica, Lubuskie: B+ (leg. det. A. Czarna, 18.04.2012); AD89 Stara Kopernia near Żagań, Lubuskie: B+ (leg. det. A. Czarna, 20.08.2009); BC28 Morzewo near Kaczory, Wielkopolskie: B+ (leg. det. A. Czarna, 30.04.2014); BC28 Piła-Leszków, Wielkopolskie: B+ (leg. det. A. Czarna, 29.04.2014); BC29 Rzadkowo near Kaczory, Wielkopolskie: B+ (leg. det. A. Czarna, 25.08.2010); BC37 Nowa Wieś Ujska near Ujście, Wielkopolskie: B1.1 (leg. det. A. Czarna, 01.05.2014); BC39 Nałęcza near Szamocin, Wielkopolskie: B+ (16.05.2006); BC39 Strzelce near Chodzież, Wielkopolskie: B2.1 (02.05.2014); BC42 Łokacz Wielki near Krzyż Wlkp., Wielkopolskie: B+ (leg. det. A. Czarna, 08.05.2013); BC45 Runowo near Trzcianka, Wielkopolskie: B+ (leg. det. A. Czarna, 21.05.2014); BC49 Radwanki near Margonin, Wielkopolskie: B+ (02.05.2014); BC77 Słonawy near Oborniki, Wielkopolskie: B1.1 (leg. det. A. Czarna, 15.09.2007); BD22 Stefanów near Zbąszyń, Wielkopolskie: B1.1 (02.05.2007); BD31 Kopanica near Siedlec, Wielkopolskie: B1.1 (18.04.2007); BC71 Mierzyn near Międzychód, Wielkopolskie: B1.1 (28.04.2010); BD70 Kiełcz near Nowa Sól, Lubuskie: B+ (leg. det. A. Czarna, 09.09.2008); BD76 Sułów near Góra, Dolnośląskie: B1.1 (04.07.2005); BD78 Dzięczyna near Poniec, Wielkopolskie: B+ (leg. det. A. Czarna, 17.05.2012); CD08 Szyszyńskie Holendry near Ślesin, Wielkopolskie: B+ (08.10.2007); CC20 Lipia Góra near Szamocin, Wielkopolskie: B+ (15.05.2006); CC27 Wypaleniska near Solec Kujawski, Kujawsko-Pomorskie: B+ (leg. det. A. Czarna,, 19.08.2008); CC36 Prądocin near Nowa Wieś Wielka, Kujawsko-Pomorskie: B1.1 (12.05.2010); CC40 Margonin, Wielkopolskie: B+ (04.09.205); CC56 Szadłogoszcz near Barcin, Kujawsko-Pomorskie: B1.1 (09.05.2008); CC59 Kijewo near Gniewkowo, Kujawsko-Pomorskie: $\mathrm{B}+$ (leg. det. A. Czarna, 18.07.2008); CC60 Runowo near Wągrowiec, Wielkopolskie: B1.1 (04.09.2005); CC64 Łaziska near Rogowo, Kujawsko-Pomorskie: B+ (26.08.2005); CC65 Parlin near Dąbrowa, Kujawsko-Pomorskie: B+ (10.05.2008); CC65 Słaboszewko near Dąbrowa, Kujawsko-Pomorskie: B+ (10.05.2008); CC69 Dąbrowa Biskupia, Kujawsko-Pomorskie: B+ (leg. det. A. Czarna, 18.07.2008); CC69 Konary near Dąbrowa Biskupia, Kujawsko-Pomorskie: B+ (leg. det. A. Czarna, 18.07.2008); CC75 Chabsko near Mogilno, Kujawsko-Pomorskie: B+ (11.05.2008); CC75 Niestronno near Mogilno, Kujawsko-Pomorskie: B+ (11.05.2008); CC76 Wszedzień near Mogilno, Kujawsko-Pomorskie: B+ (10.05.2008); CC81 Turostowo near Kiszkowo, Wielkopolskie: B1.1 (16.08.2005); BE08 Prusice, Dolnośląskie: B+ (leg. det. A. Czarna, 08.09.2009); CC92 Wierzyce near Łubowo, Wielkopolskie: B2.2 (08.08.2005); CD00 Łowęcin near Swarzędz, Wielkopolskie: B+ (leg. det. A. Czarna, 14.08.2008); CD10 Kleszczewo, Wielkopolskie (leg. det. A. Czarna, 11.06.2005); CD30 Gumienice near Pogorzela, Wielkopolskie: B1.1 (01.06.2005); CD35 Michalinów Oleśnicki near Zagórów, Wielkopolskie: B+ (16.05.2005); CD46 Stare Grądy near Grodziec, Wielkopolskie: B1.1 (29.09.2007); CD46 Wielołęka, near Grodziec, Wielkopolskie: B+ (29.09.2007); CD50 Borek Wlkp., Wielkopolskie: B+ (13.06.2005); CC84 Dąbrowa near Trzemeszno, Wielkopolskie: B2.2 (leg. det. A. Czarna, 11.05.2008); CD90 Ludwikowo near Bytoń, Kujawsko-Pomorskie: B1.1 (leg. det. A. Czarna, 14.06.2008); CE13 Ligota Rybińska near Międzybórz, Dolnośląskie: B+ (leg. det. A. Czarna, 08.08.2010); DC40 Brzoza near Wielka Nieszawka, Kujawsko-Pomorskie: B1.1 (leg. det. A. Czarna, 18.07.2008); DD02 Cetty near Chodecz, Kujawsko-Pomorskie: B+ (leg. det. A. Czarna, 18.06.2008); DD30 Daniszew near Kościelec, Wielkopolskie: B1.1 (27.09.2007).

Rosa multiflora Thunb. (leg. det. A. Czarna)

AD47 Leśniów Wlk. near Czerwieńsk, Lubuskie: B+ (27.08.2008); BC51 Drezdenko, Lubuskie: B+ (02.10.2013); BD95 Kwielice near Grębocice, Dolnośląskie: B+ (31.08.2009); CD49 Turek, Wielkopolskie: $\mathrm{B}+(28.09 .2007)$. 
Rosa palustris Marshall (leg. A. Czarna, det. J. Zieliński)

AD65 Chocicz near Lubsko, Lubuskie: B+ (03.04.2014).

Rosa 'Poznań'(leg. A. Czarna, det. J. Zieliński)

BD08 Poznań, Wielkopolskie: B+ (30.10.2013).

Rosa rugosa Thunb. (leg. A. Czarna, det. J. Zieliński)

AD42 Piaski, Lubuskie: B+ (27.08.2012); AD65 Chocicz near Lubsko, Lubuskie: B+ (21.09.2008); AD86 Kadłubia near Żary, Lubuskie: B+ (24.09.2009); BC17 Piła, Wielkopolskie: B+ (24.08.2010); BC27 Ługi Ujskie near Ujście, Wielkopolskie: B+ (07.09.2011); BC27 Piła, Wielkopolskie: B+ (29.04.2014); BC46 Bukowiec near Czarnków, Wielkopolskie: B+ (04.09.2011); BC76 Szamotuły, Wielkopolskie: B+ (08.05.2007); BD56 Żakowo near Lipno, Wielkopolskie: B1.1 (20.06.2006); CC27 Bydgoszcz-Fordon, Kujawsko-Pomorskie, B+ (21.03.2015); CC48 Glinno Wlk. near Rojewo, Kujawsko-Pomorskie: B+ (17.08.2008); CC48 Jaszczołtowo near Rojewo, Kujawsko-Pomorskie: B+ (17.08.2008); CD49 Turek, Wielkopolskie: B+ (28.09.2007); CE01 Milicz, Dolnośląskie: $\mathrm{B}+(17.06 .2005)$.

Rosa rugosa Thunb. $\times$ R. davurica Pall. (leg. A. Czarna, det. J. Zieliński)

BC50 Błotno near Zwierzyn, Lubuskie: B+ (07.05.2013).

Rosa rugosa Thunb. $\times$ R. majalis (leg. A. Czarna, det. J. Zieliński)

BC17 Piła, Wielkopolskie: B+ (24.08.2010); BC51 Pełcza near Drawsko, Wielkopolskie: B+ (10.08.2006).

Rosa xrugotida Darthuis (leg. A. Czarna, det. J. Zieliński)

BD09 Poznań, Wielkopolskie: B1.1 (04.06.2012); DD20 Koło, Wielkopolskie: B+ (26.06.2012).

Rosa xspaethiana Graebn. (leg. A. Czarna, det. J. Zieliński)

AD18 Mostki near Lubrza, Lubuskie: B1.1 (09.05.2010); AD43 Budoradz near Gubin, Lubuskie: B+ (14.06.2010); BC51 Pełcza near Drawsko, Wielkopolskie: B+ (10.08.2006); CC48 Glinno Wielkie near Rojewo, Kujawsko-Pomorskie: B+ (17.08.2008).

Rosa spinosissima 'Nana' (leg. det. A. Czarna, with few exceptions)

AC89 Twierdzielewo near Przytoczna, Lubuskie: B1.1 (08.09.2006); AD24 Cybinka, Lubuskie: B+ (26.09.2006); AD44 Drzeńsk Wielki near Gubin, Lubuskie: B+ (14.06.2010); AD53 Bieżyce near Gubin, Lubuskie: B+ (18.09.2008); AD57 Drzonów near Świdnica, Lubuskie: B1.1 (02.04.2014); BC01 Trzciel, Lubuskie: B1.1 (21.04.2010); BC18 Brodna near Kaczory, Wielkopolskie: B1.1 (26.08.2010); BC36 Teresin near Trzcianka, Wielkopolskie: B+ (12.05.2014); BC61 Lubiatów near Drezdenko, Lubuskie: B2.2 (26.04.2010); BD04 Jastrzębniki near Opalenica, Wielkopolskie: B+ (15.04.2010); CC20 Lipia Góra near Szamocin, Wielkopolskie: B+ (leg. A. Czarna, det. J. Zieliński, 15.05.2006); CC22 Kowalewko near Kcynia, Kujawsko-Pomorskie: B+ (23.09.2011); CC25 Przyłęki near Białe Błota, Kujawsko-Pomorskie: B+ (22.08.2008); CC26 Prądocin near Nowa Wieś Wielka, Kujawsko-Pomorskie: B+ (21.08.2008); CC36 Bydgoszcz, Kujawsko-Pomorskie: B1.1 (20.08.2008); CC48 Glinno Wielkie near Rojewo, Kujawsko-Pomotskie: B+ (17.08.2008); CC48 Osieczek near Rojewo, Kujawsko-Pomorskie: B2.2 (17.08.2008); CC48 Osiek near Rojewo, Kujawsko-Pomorskie: B+ (17.08.2008); CC60 Runowo near Wągrowiec, Wielkopolskie: B1.1 
(leg. A. Czarna, det. J. Zieliński, 04.09.2005); CC76 Dębina near Janikowo, Kujawsko-Pomorskie: B1.1 (10.10.2007); CC76 Szczeglin near Mogilno, Kujawsko-Pomorskie: B+ (09.05.2008); CD92 Joachimówka near Milicz, Dolnośląskie: B+ (31.07.2010).

Rosa virginiana Mill. (leg. A. Czarna, det. J. Zieliński)

CC33 Słonawy near Szamocin, Wielkopolskie: B+ (10.05.2006).

\section{RESULTS}

As a result of floristic research in 2,169 old, neglected cemeteries in Wielkopolska, 23 species and 12 interspecific hybrids of the genus Rosa have been identified (Table 1). These include 14 native taxa: Rosa agrestis Savi, $R$. canina L., $R$. canina L. $\times R$. glauca Pourret, $R$. canina L. $\times R$. rubiginosa L., $R$. dumalis Bechst., $R$. inodora Fries, $R$. inodora Fries $\times R$. rubiginosa L., $R$. jundzillii Besser, $R$. rubiginosa L., $R$. rubiginosa $\times R$. sherardii Davies, $R$. sherardii Davies, $R$. tomentosa Smith, $R$. villosa $\mathrm{L}$, and $R$. zalana Wiesb. The 21 alien taxa are represented by Rosa xalba L., $R$. blanda Ait., $R$. xcentifolia L., $R$. xdamascena Mill., $R$. foetida J. Herrmann, $R$. foliolosa Nutt., $R$. xfrancofourtana Muenchh., $R$. gallica L. 'Plena', $R$. glauca Pourret, $R$. gorenkensis Besser, $R$. majalis J. Herrmann 'Foecundissima', $R$. multiflora Thunb., $R$. palustris Marshall, $R$. palustris Marshall $\times R$. rugosa Thunb., $R$. 'Poznań, $R$. rugosa Thunb., $R$. rugosa Thunb. $\times R$. davurica Pall., $R$. rugosa Thunb. $\times R$. majalis J. Herrmann, $R$. xrugotida Darthuis, $R$. xspaethiana Graebn., $R$. spinosissima 'Nana', and $R$. virginiana Mill. Some of them appeared there probably spontaneously, while others are relics of their cultivation in the cemeteries in the past.

The most frequent spontaneously occurring species was $R$. canina, recorded in 772 cemeteries. Another frequent species was $R$. sherardii, recorded in $60 \mathrm{cem}-$ eteries, followed by $R$. dumalis (19) and $R$. rubiginosa (18). The rarest taxa were $R$. agrestis, $R$. canina $\times R$. glauca, $R$. canina $\mathrm{L} . \times R$. rubiginosa, $R$. inodora, $R$. inodora $\times R$. rubiginosa, $R$. jundzillii, $R$. rubiginosa $\times R$. sherardii, $R$. tomentosa, and $R$. villosa, represented in this study by single localities. It is noteworthy that 12 of the taxa are hybrids, both deriving from cultivation (7) and spontaneous (5).

The most frequent alien taxa were Rosa gallica L. 'Plena' (80 localities), $R$. xfrancofourtana (72), R. majalis (55), Rosa spinosissima L. 'Nana' (22), and $R$. rugosa (15). All the taxa mentioned above reached high values of cover-abundance in the cemeteries because they produce stolons. All of them were doubleflowered.

In comparison with Zieliński's monograph (17), five of the taxa recorded in cemeteries are new to the Polish flora: $R$. foliolosa, $R$. 'Poznan', $R$. rugosa $\times$ $R$. majalis, $R$. rugosa $\times R$. davurica, $R$. rugotida, and $R$. xspaethiana. 
Pobrane z czasopisma Annales C - Biologia http://biologia.annales.umcs.pl

Data: 26/04/2023 15:26:43

\section{DISCUSSION}

The 35 taxa of the genus Rosa recorded in old cemeteries in Wielkopolska include 14 native species and 21 alien taxa, some of them being hybrids. In Zieliński's (16) report from southern Wielkopolska, as well as from the Lubusz

Table 1. Number of localities of the recorded taxa in old cemeteries in Wielkopolska

\begin{tabular}{|c|c|c|}
\hline No. & Taxon & No. of localities \\
\hline 1 & Rosa agrestis Savi & 1 \\
\hline 2 & Rosa xalba L. 'Plena' & 18 \\
\hline 3 & Rosa blanda Ait. & 2 \\
\hline 4 & Rosa canina $\mathrm{L}$. & 768 \\
\hline 5 & Rosa canina L. $\times$ R. glauca Pourret & 1 \\
\hline 6 & Rosa canina $\mathrm{L} . \times$ R. rubiginosa $\mathrm{L}$. & 1 \\
\hline 7 & Rosa xcentifolia $\mathrm{L}$. & 7 \\
\hline 8 & Rosa xdamascena Mill. & 6 \\
\hline 9 & Rosa dumalis Bechst. & 19 \\
\hline 10 & Rosa foetida J. Herrmann & 2 \\
\hline 11 & Rosa foliolosa Nutt. & 1 \\
\hline 12 & Rosa xfrancofourtana Muenchh. & 72 \\
\hline 13 & Rosa gallica L. 'Plena' & 80 \\
\hline 14 & Rosa glauca Pourret & 1 \\
\hline 15 & Rosa gorenkensis Besser & 4 \\
\hline 16 & Rosa inodora Fries & 1 \\
\hline 17 & Rosa inodora Fries $\times$ R. rubiginosa $\mathrm{L}$. & 1 \\
\hline 18 & Rosa jundzillii Besser & 1 \\
\hline 19 & Rosa majalis J. Herrmann 'Foecundissima' & 55 \\
\hline 20 & Rosa multiflora Thunb. & 4 \\
\hline 21 & Rosa palustris Marshall & 1 \\
\hline 22 & Rosa 'Poznań' & 1 \\
\hline 23 & Rosa rubiginosa $\times R$. sherardii Davies & 1 \\
\hline 24 & Rosa rubiginosa $\mathrm{L}$. & 18 \\
\hline 25 & Rosa rugosa Thunb. & 15 \\
\hline 26 & Rosa rugosa Thunb. $\times$ R. majalis J. Herrmann & 2 \\
\hline 27 & Rosa rugosa Thunb. $\times$ R. davurica Pall. & 1 \\
\hline 28 & Rosa xrugotida Darthuis & 2 \\
\hline
\end{tabular}


Pobrane z czasopisma Annales C - Biologia http://biologia.annales.umcs.pl

Data: 26/04/2023 15:26:43

ROSES (ROSA SPP.) IN OLD CEMETERIES IN THE WIELKOPOLSKA REGION (W POLAND)

\begin{tabular}{|c|l|c|}
\hline 29 & Rosa sherardii Davies & 60 \\
\hline 30 & Rosa xspaethiana Graebn. & 4 \\
\hline 31 & Rosa spinosissima L. 'Nana' & 22 \\
\hline 32 & Rosa tomentosa Smith & 1 \\
\hline 33 & Rosa villosa L. & 6 \\
\hline 34 & Rosa virginiana Mill. & 1 \\
\hline 35 & Rosa zalana Wiesb. & 1 \\
\hline
\end{tabular}

Land (Ziemia Lubuska) (15), 12 native species were recorded. Stefanek (11) reported 10 native species from the Wielkopolska Lakeland. In total for Poland, Zieliński (17) listed 14 native species, but without any hybrid taxa, and the same number of alien species. Sobisz and Antkowiak (10), in 43 cemeteries in the Słowiński National Park, found only six species of the genus Rosa: $R$. canina, $R$. dumalis, $R$. gallica, $R$. multiflora, $R$. rubiginosa, and $R$. rugosa. It is surprising that $R$. sherardii was lacking there. Galera et al. (2) listed four taxa from the eastern part of Warsaw: R. multiflora, $R$. glauca, $R$. rugosa, and $R$. multiflora $\times R$. sp. In the Masurian Landscape Park, only Rosa canina was recorded (6). In selected cemeteries in the town of Ostrów Wielkopolski (1), only two species were noted, $R$. canina and $R$. rubiginosa, and each of them was present in only a single cemetery. One species, $R$. rubiginosa, was also recorded in selected cemeteries of Lublin and its environs (12), while all the other specimens were not identified. Rose identification is difficult, so they need to be collected and identified by a specialist.

In the study area, the four most common species of the genus Rosa were $R$. canina, $R$. sherardii, $R$. dumalis, and $R$. rubiginosa. By contrast, in Stefanek's study (11), $R$. dumalis ranked second, while the order of the remaining common roses was the same. However, in Zieliński's (16) report, the most common rose was $R$. sherardii, followed by $R$. dumalis, $R$. canina, and even rarer $R$. rubiginosa.

The high number of roses found in the old cemeteries in Wielkopolska may result from their location, often in slightly elevated areas, as well as dense shrub communities and tall herb communities developed at their edges. These were often rose thickets, difficult to penetrate. They were usually composed of a single species, less commonly of several species. In the Dynów Foothills (Pogórze Dynowskie), Rosa canina was found on grassy field borders, roadsides, wastelands, at the edges of forests and shrub communities, while $R$. sherardii on dry hills, in thickets, and on roadsides (3).

The alien taxa that produced fully ripe fruits, include Rosa xalba, $R$. foliolosa, $R$. multiflora, and $R$. rugosa. The last two are classified as kenophytes in Poland (14). 
Old, forgotten rose species and varieties should be popularized in modern cemeteries and home gardens. They are characterized by a high ornamental value because of their beautiful appearance and abundant blossoms, as well as pleasant smell. The symbolic meaning of historical roses, although often not understood by people today, has contributed to creation of the specific atmosphere of cemeteries.

\section{REFERENCES}

1. Celka Z., Żywica J., 2004. Flora naczyniowa wybranych cmentarzy Ostrowa Wielkopolskiego i okolic. Roczniki Akademii Rolniczej w Poznaniu 343, Bot. 7: 11-31.

2. Galera H., Sudnik-Wójcikowska, Lisowska M., 1993. Flora cmentarzy lewobrzeżnej Warszawy na tle flory miasta. Fragm. Flor. Geobot. 38(1): 237-261.

3. Gutkowska B., Niedźwiecka J., 2014. Rodzaj Rosa (Rosaceae) na Pogórzu Dynowskim (SE Polska). Fragm. Florist. Geobot. Polon. 21(2): 217-227.

4. Hagen Th., 2006. Róże. Portrety roślin, wybór, pielęgnacja. Muza SA, Warszawa, Poland, pp. 95.

5. Henschke M., 2010. Róże. Działkowiec, Warszawa, Poland, pp. 72.

6. Hołdyński Cz., Żurkowska T., 2001. Drzewa i krzewy opuszczonych cmentarzy w Mazurskim Parku Krajobrazowym. Rocznik Dendrologiczny, vol. 49: 265-273.

7. Popek R., 2002. Róże dziko rosnące Polski. Klucz-Atlas. Plantpress, Kraków, Poland, pp. 112.

8. Popek R., 2007. Dziko rosnące róże Europy. Officina Botanica, ATUS, Kraków, Poland, pp. 119.

9. Richter G., 1995. Kryteria planowania zieleni na cmentarzach. [In:] O. Czerner, I. Juskiewicz, Sztuka cmentarna, ICOMOS. Polish National Committee, Museum of Art, Wrocław, Poland.

10. Sobisz Z., Antkowiak W., 2009. Flora naczyniowa cmentarzy na obszarze Słowińskiego Parku Narodowego. Słupskie Prace Biologiczne 6: 127-144.

11. Stefanek W., 1984 (1986). Róże na Pojezierzu Wielkopolskim, między Wartą a Wisłą. Fragm. Flor. Geobot. 30(3): 171-184.

12. Trzaskowska E., Karczmarz K., 2013. Spontaneous vascular flora of selected cemeteries in Lublin and the surrounding area. Acta Agrobotanica 66(2): 107-122.

13. Zając A., 1978. Założenia metodyczne „Atlasu rozmieszczenia roślin naczyniowych w Polsce”. Wiadomości Botaniczne 22(3): 145-150.

14. Zając A., Zając M., Tokarska-Guzik B., 1998. Kenophytes in the flora of Poland: list, status and orgin. Phytocoenosis, vol. 10 (N.S.), Supplementum Cartographiae Geobotanicae 9: 107-116, Warszawa-Białowieża, Poland.

15. Zieliński J., 1977. Róże Wysoczyzny Lubuskiej. Fragm. Flor. Geobot. 23(2): 125-140.

16. Zieliński J., 1980. Rozmieszczenie róż w południowej Wielkopolsce. Fragm. Flor. Geobot, 26(1): 53-64.

17. Zieliński J., 1987. Rodzaj Rosa L. [In:] A. Jasiewicz (red.) Flora Polski, Rośliny naczyniowe 5, pp. 49. Państwowe Wydawnictwo Naukowe, Warszawa-Kraków, Poland.

18. Żukowski W., Celka Z., Chmiel J., Jackowiak B., Latowski K., Szkudlarz P., 2001. Rozmieszczenie wybranych gatunków roślin ginących w Wielkopolsce. Bogucki Wydawnictwo Naukowe, Poznań, Poland, pp. 68.

19. Internet 1. http://poradnikogrodniczy.pl/rosliny_na_cmentarzu.php, pozyskano 20.12.2014 
Pobrane z czasopisma Annales C - Biologia http://biologia.annales.umcs.pl

Data: 26/04/2023 15:26:43

ROSES (ROSA SPP.) IN OLD CEMETERIES IN THE WIELKOPOLSKA REGION (W POLAND)

\section{ACKNOWLEDGEMENTS}

I would like to thank Prof. Dr hab. Jerzy Zieliński (Institute of Dendrology in Kórnik) for verification as well as identification of the collected herbarium specimens and rose collections. I am also grateful to Dr hab. Wojciech Antkowiak for help in creating the rose collection. The study was supported to a large extent by the National Science Centre (grant number NN 304204 937). The manuscript was partly translated into English by Sylwia Ufnalska. 